\title{
Towards simulation of elasto-plastic deformation: An investigation
}

\author{
ARUN R RAO and U SHRINIVASA \\ Department of Mechanical Engineering, Indian Institute of Science, \\ Bangalore 560 012, India \\ e-mail: udipi@mecheng.iisc.ernet.in
}

MS received 5 December 2001

\begin{abstract}
Can the deformation of a solid body during plastic flow be assumed to be similar to that of fluids? Here we investigate the possibility of using a modified Navier-Stokes equation as the governing differential equation by including elastic resistance. We adopt the microscopic point of view to explain the material behaviour by laying special emphasis on strain localisation and tension instabilities. A spring and damper model is constructed to obtain approximate simulation of the material behaviour. Based upon the understanding developed from simulating simple tests, we re-formulate the field equation using resistances to change in volume and shape. The new field equation reduces to the Navier-Stokes equation in the fluid limit and Cauchy's equation in the solid limit. The viscosity and second viscosity of fluids are clearly defined. Bulk and shear modulii and solid damping determine the solid behaviour. Pressure disappears from the field equation and so there is no need to invoke the continuity equation. The four material parameters are determinable from simple measurments. This paper tries to capture the various steps of the investigation which lead to the final result.
\end{abstract}

Keywords. Elasto-plastic deformation; modified Navier-Stokes equation; strain localisation; tension instability; material behaviour; Cauchy's equation.

\section{Introduction}

The mathematical theory of plasticity has been in use for many years but the formulation of a field equation for simulation of elasto-plastic deformation is still a matter of discussion. Several engineering idealised theories are proposed but they fail to account for the various phenomena observed. Due to the complicated nature of the plasticity problem, theory in earlier days is chiefly confined to small deformation, viz. infinitesimal theory with some special emphasis on hardening laws and, some special material responses (Hill 1950; Prager \& Hodge 1951; Prager 1955; Drucker 1960; Naghdi 1960). These theories are 'rate-independent' to cover some general class of materials with consideration of a few special properties such as material anisotropy, creep and thermal effects.

A large amount of work is carried out by different schools of plasticity in the area of finite deformation either in the Lagrangian or in the Eulerian coordinate systems using stress-based 
or strain-based approaches. The stress-based formulation (Green \& Naghdi 1965, 1966) is in turn found inadequate (Naghdi \& Trapping 1975) to explain some material properties and is non-trivial for implementing numerically. Later Naghdi and co-workers developed strainbased formulation (Naghdi \& Trapp 1975; Casey \& Naghdi 1981, 1983) to overcome these difficulties and made it suitable to solve numerically.

In the literature, we can often see the development of the constitutive equations for deformation theories on macroscopic scale (Barbe et al 2001). But the experimental observations made by Taylor \& Elam $(1923,1925,1926)$ reveals the importance of microscopic structure during large deformation. These observations led further to development of various microscopic theories (Batdorf \& Budiansky 1949; Koiter 1953; Kröner 1960; Budiansky \& Wu 1962; Hill 1965, 1966; Hutchinson 1970; Rice 1976; Bazant 1984) and more recently to those of Qui \& Weng (1992, 1995), Rubin (1996), Acharya \& Beaudoin (2000), Bigoni et al (2000), and Acharya (2001). All these workers tried to develop the theory by considering various aspects of microstructure like single crystal or polycrystal deformation, strain localisation, material anisotropy etc. Nowadays, these microstructure theories are covering more and more ground as the physics of micro-mechanics is being deeply studied and understood (Bertram 1998) to provide answers to most of the questions.

With the development of theory, efforts are made to develop the plasticity theory in the Eulerian coordinate system as it is found more suitable for large deformations. Kröner (1960) initiates work in Eulerian coordinate system and later it is carried out by the others (Backman 1964; Lee \& Liu 1967; Lee 1969; Dienes 1979, 1986; Lee et al 1983; Loret 1983; NematNasser 1983; Dafalias 1983, 1984; Atluri 1984; Agah-Tehrani et al 1987). In recent years we see some schools of plasticity use the 'arbitrary Lagrangian-Eulerian' description, which combines both Lagrangian and Eulerian descriptions to solve the problems (Haber 1984; Huetink et al 1990; Ghosh \& Suresh 1996; Braess \& Wriggers, 2000).

Most of the existing plasticity theories depend heavily upon yield function to differentiate between elastic and plastic regions (Hill 1994). Yield function is dependent upon stress, plastic strain and work hardening parameters and is usually determined by experiments. However, the existence of the yield surface or yield condition is questionable. Some investigators (Seth 1963, 1964; Valanis 1971, 1975; Bodner \& Partan 1975; Lee \& Zaverl 1978, 1979; Stouffer \& Bodner 1979) lay special emphasis on the possibility of developing the theory of plasticity without introducing a yield surface or yield condition.

A body is understood to deform simultaneously by elastic and plastic strains under the application of external load. In the classical theory of elasticity the linear trend of infinitesimal deformation is expressed by the generalised Hooke's law. In actuality, the cyclic loaddeformation or stress-strain curve is not a single-valued function but forms a hysteretic loop (Lazan 1968). Lazan (1968) also observed that all materials, composites and structural assemblies do not behave in a perfectly elastic manner even at very low stresses. Inelasticity is always present under all types of loading. Hence the constitutive equations for the elastic and the plastic parts of the deformation cannot be treated independently. Elastic and plastic effects should dovetail into each other even when elastic behaviour is assumed to be unaffected by the preceding plastic deformations. This supposition avoids any assumption of yield condition or yield surface to determine any elastic-plastic boundary, which may or may not exist (Seth 1970) and is sometime also ambiguous in multi-axial cases (Chen \& Lu 2000).

Existing plasticity theory is based on certain experimental observations on the macroscopic behaviour of metals in the uniform state of combined stresses (Chakrabarty 1998). These observed results are then idealised into a mathematical formulation to describe the behaviour of metals under complex stresses. However a lot of assumptions are involved to simplify the 
complex phenomenon of plastic deformation. These assumptions make the theory incapable of explaining various material phenomenon such as the Bauschinger effect, anisotropy, time and temperature effects, size effect, stress inhomogeneity, material damping etc.

Thus, existing models are able to explain some of the phenomenon observed, but leave behind a lot of controversies. Each model is useful for a particular purpose with some welldefined boundary conditions. However, many phenomena occurring during plastic deformation remain unexplained. Hence, there is an urgent need for a constitutive equation which in general will correctly explain deformation behaviour and also the physics involved in the deformation process. Therefore we try to develop a field equation from first principles (as against from experimental observations) which assumes a generalised relation between stress, strain and strain rate that could represent the material behaviour in the elastic range, transition from the elastic to plastic state and in the plastic range, and later use the same to explain most of the phenomena observed in practice.

\section{Formulation}

Zienkiewicz \& Godbole (1975) pointed out that in metals, plasticity with large deformation, whose elastic strains are negligible, stands on the borderline of fluid and solid mechanics. Under equilibrium conditions both solid and fluid are identical provided dynamic forces are neglected. Thus one can conclude that the solution of a viscous flow problem is identical to the solution of an equivalent incompressible elastic problem in which displacements are interpreted as velocities and infinitesimal strain as strain rate (Zienkiewicz \& Godbole 1974). Thus all the techniques available in the literature for the solution of one problem are applicable to the other. The analogy that in the plastic state metals behave like viscous fluids helps to express the formulation in the Eulerian coordinate system which seems more relevant in large elastic-plastic deformation (Prager 1961). Further we see that in recent and current literature, most plasticity theories are inclined towards an Eulerian formulation in stress space setting. This is mainly because of its analogy with viscous fluid flow, the construction of the theory in terms of Cauchy stresses and its rate being more fundamental. Cauchy's stress tensor $\boldsymbol{P}$ is defined in the current configuration as,

$$
\boldsymbol{P}=\tau_{i j} e_{i} \otimes e_{j}
$$

where $\tau_{i j}$ are the stress components and $e_{i}$ and $e_{j}$ are the unit co-ordinate vectors.

\subsection{Contribution from the strain-rate tensor}

Every material displays more or less viscous properties. For many important structural materials, these properties appear to surface after the plastic state has been reached. It is normally believed that materials display viscous properties only in the plastic range. We however look for a universal model.

Consider the stress (following Lamb 1945) exerted across any small area situated at a point $\mathrm{O}$ in the continuum (figure 1). The deviation of the state of stress denoted by $p_{x x}, p_{x y}, \ldots$ from one of pressure uniform in all directions depends entirely on the motion of distortion (i.e. on instantaneous rate of deformation) in the neighbourhood of O, i.e. on 6 quantities $a, b, c, f, g, h$ which are defined as,

$$
\begin{aligned}
& a=\partial \dot{u} / \partial x, \quad b=\partial \dot{v} / \partial y, \quad c=\partial \dot{w} / \partial z, \\
& f=\frac{\partial \dot{w}}{\partial y}+\frac{\partial \dot{v}}{\partial z}, \quad g=\frac{\partial \dot{u}}{\partial z}+\frac{\partial \dot{w}}{\partial x}, \quad h=\frac{\partial \dot{v}}{\partial x}+\frac{\partial \dot{u}}{\partial y},
\end{aligned}
$$




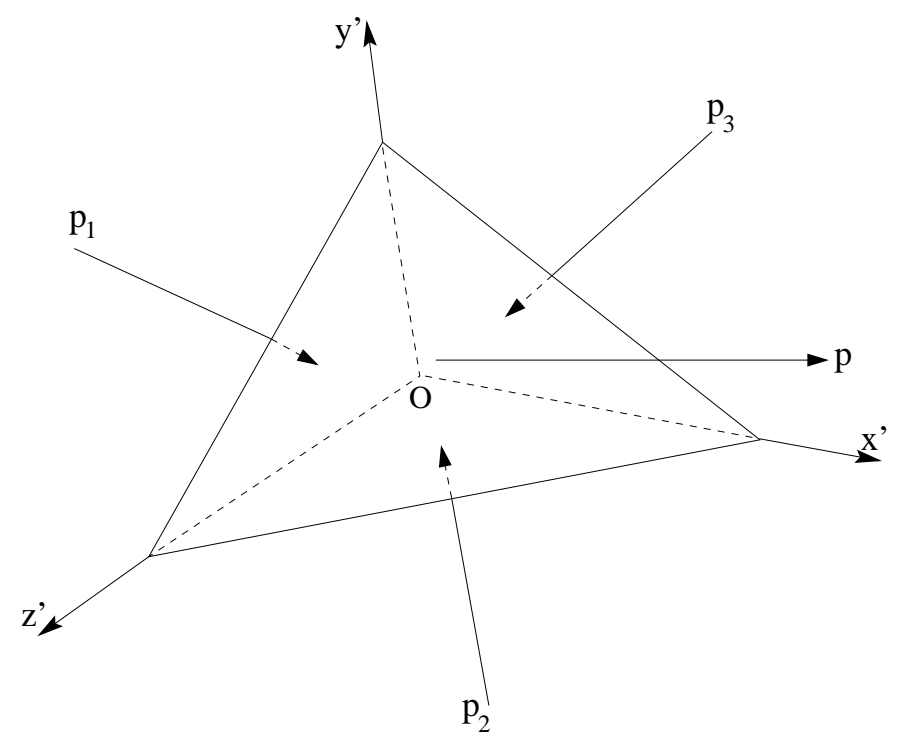

Figure 1. Force equilibrium for a point in fluid.

and, the rate of the deformation matrix $A$, turns out to be

$$
\boldsymbol{A}=\left(\begin{array}{ccc}
a & h / 2 & g / 2 \\
h / 2 & b & f / 2 \\
g / 2 & f / 2 & c
\end{array}\right)
$$

We neglect here the rotation tensor because the continuum rotation due to deformation is real but irrelevant as it does not contribute to stresses (Drucker 1988). In other words, "the plastic spin nullifies the continuum spin" (Dafalias 1985).

Let $a^{\prime}, b^{\prime}, c^{\prime}$ be the rates of extension along principal axes of distortion. Let $p_{1}, p_{2}, p_{3}$ be the principal stresses exerted at $\mathrm{O}$ across the planes $y^{\prime} z^{\prime}, z^{\prime} x^{\prime}, x^{\prime} y^{\prime}$ and wholly perpendicular to these planes and $p$ be the mean stress, which is the arithmetic mean of the normal pressure on any three mutually perpendicular planes through the point $\mathrm{O}$.

Now $p_{1}, p_{2}, p_{3}$ differ from $-p$ by quantities depending on the matrix of distortion, which must be functions of $a^{\prime}, b^{\prime}, c^{\prime}$ only. The hypothesis made in the classical theory is that these functions are linear, i.e. they depend on the first invariant of the rate of deformation tensor (Lamb 1945). Considering however the remaining two invariants also of the same tensor, we can write (following Anjaneyulu 1999),

$$
\begin{aligned}
& p_{1}=-p+\lambda_{1}\left(a^{\prime}+b^{\prime}+c^{\prime}\right)+\lambda_{2} a^{\prime}+\lambda_{3} a^{\prime} b^{\prime}+\lambda_{4}\left(a^{\prime} b^{\prime}+b^{\prime} c^{\prime}+c^{\prime} a^{\prime}\right)+\lambda_{5} a^{\prime} b^{\prime} c^{\prime}, \\
& p_{2}=-p+\lambda_{1}\left(a^{\prime}+b^{\prime}+c^{\prime}\right)+\lambda_{2} b^{\prime}+\lambda_{3} b^{\prime} c^{\prime}+\lambda_{4}\left(a^{\prime} b^{\prime}+b^{\prime} c^{\prime}+c^{\prime} a^{\prime}\right)+\lambda_{5} a^{\prime} b^{\prime} c^{\prime}, \\
& p_{3}=-p+\lambda_{1}\left(a^{\prime}+b^{\prime}+c^{\prime}\right)+\lambda_{2} c^{\prime}+\lambda_{3} c^{\prime} a^{\prime}+\lambda_{4}\left(a^{\prime} b^{\prime}+b^{\prime} c^{\prime}+c^{\prime} a^{\prime}\right)+\lambda_{5} a^{\prime} b^{\prime} c^{\prime} .
\end{aligned}
$$

Adding these three equations and using the definition of $p$, i.e., $p=\left(p_{1}+p_{2}+p_{3}\right) / 3$ we can solve for the constants. Thus the resistance part due to $p$ and the strain-rate tensor in the 
principal coordinates become,

$$
\boldsymbol{P}_{d}^{\prime}=-p \boldsymbol{I}+\lambda_{1} J_{1}^{\prime} \boldsymbol{I}-3 \lambda_{1}\left(\begin{array}{ccc}
a^{\prime} & 0 & 0 \\
0 & b^{\prime} & 0 \\
0 & 0 & c^{\prime}
\end{array}\right)-3 \lambda_{4} J_{3}^{\prime}\left(\begin{array}{ccc}
a^{\prime} & 0 & 0 \\
0 & b^{\prime} & 0 \\
0 & 0 & c^{\prime}
\end{array}\right)^{-1}+\lambda_{4} J_{2}^{\prime} \boldsymbol{I},
$$

where $J_{1}^{\prime}, J_{2}^{\prime}$ and $J_{3}^{\prime}$ are the first, second and third invariants of strain rate tensors in the principal coordinates system. By transforming $\boldsymbol{P}_{d}^{\prime}$ in principal coordinates to the current coordinates, we get,

$$
\begin{aligned}
\boldsymbol{P}_{\boldsymbol{d}} & =\boldsymbol{L}^{\boldsymbol{T}} \boldsymbol{P}_{\boldsymbol{d}}^{\prime} \boldsymbol{L} \\
& =-p \boldsymbol{I}+\lambda_{1}\left(J_{1} \boldsymbol{I}-3 \boldsymbol{A}\right)+\lambda_{4}\left(J_{2} \boldsymbol{I}-3 \operatorname{Adj}(\boldsymbol{A})\right) .
\end{aligned}
$$

Here $\lambda_{1}$ and $\lambda_{4}$ are the unknown material constants and $J_{1}, J_{2}$ are the first two invariants of the strain rate tensor in the current coordinate system.

\subsection{Contribution from the strain tensor}

In classical elasticity theory, stresses are assumed to be linear functions of strains, and are given by the generalised Hooke's law. Anjaneyulu (1999) considers all the three invariants of the strain tensor, to obtain a generalised relation as,

$$
\boldsymbol{P}_{e}=\lambda I_{1} \boldsymbol{I}+2 \mu \boldsymbol{E}+\eta_{1} \operatorname{Adj}(\boldsymbol{E})+\eta_{2} I_{2} \boldsymbol{I}+\eta_{3} I_{3} \boldsymbol{I},
$$

where $\boldsymbol{E}$ is the small strain tensor,

$$
\begin{aligned}
\boldsymbol{E} & =\left[\begin{array}{ccc}
\epsilon_{x x} & \epsilon_{x y} & \epsilon_{x z} \\
\epsilon_{y x} & \epsilon_{y y} & \epsilon_{y z} \\
\epsilon_{z x} & \epsilon_{z y} & \epsilon_{z z}
\end{array}\right], & & \\
\epsilon_{x x} & =\partial u / \partial x, & \epsilon_{y y}=\partial v / \partial y, & \epsilon_{z z}=\partial w / \partial z, \\
\epsilon_{x y} & =\epsilon_{y x}=\frac{\partial u}{\partial y}+\frac{\partial v}{\partial x}, & \epsilon_{y z}=\epsilon_{z y}=\frac{\partial v}{\partial z}+\frac{\partial w}{\partial y}, & \epsilon_{z x}=\epsilon_{x z}=\frac{\partial w}{\partial x}+\frac{\partial u}{\partial z},
\end{aligned}
$$

where $\lambda$ and $\mu$ are Lame's constants, $\eta_{1}, \eta_{2}, \eta_{3}$ are unknown material constants and $I_{1}, I_{2}$ and $I_{3}$ are the first, second and third invariants of strain in the current coordinate system. Since plastic flow is usually associated with large deformation gradients, we consider it appropriate to use the Almansi strain tensor, which in spatial form is given by,

$$
E_{i j}^{*}=\frac{1}{2}\left[\frac{\partial u_{i}}{\partial x_{j}}+\frac{\partial u_{j}}{\partial x_{i}}-\frac{\partial u_{k}}{\partial x_{i}} \frac{\partial u_{k}}{\partial x_{j}}\right]
$$

The above strain tensor $\boldsymbol{E}^{*}$ is an exact one in the Eulerian coordinate system and no approximations are involved (Fung 1965). While using the expression for $\boldsymbol{P}_{\boldsymbol{e}}$ there is no explicit reference to the original undeformed state. We need only the gradients of $\boldsymbol{P}_{\boldsymbol{e}}$ to set up the equations of motions in the next section.

It is commonly accepted in practice that, during elasto-plastic flow, instantaneous unloading is always linearly elastic to begin with. This is difficult if $\eta_{1}, \eta_{2}$ and $\eta_{3}$ have non-zero 
values. Therefore to be consistent with observations particularly on steels, we assume that the constant $\eta_{1}=\eta_{2}=\eta_{3}=0$, which reduces the expression for $\boldsymbol{P}_{\boldsymbol{e}}$ to,

$$
\boldsymbol{P}_{\boldsymbol{e}}=\lambda K_{1} \boldsymbol{I}+2 \mu \boldsymbol{E}^{*}
$$

where $K_{1}=E_{k k}^{*}$.

\subsection{Final form of the equations of motion}

The final form of the equation of motion can be obtained by substituting the contributions from strain and strain rate tensors in the equation for the law of balance of linear momentum in spatial (Eulerian) form (Malvern 1969),

$$
\rho \frac{D \dot{\mathbf{U}}}{D t}=\rho \mathbf{B}+\left(\nabla \cdot \boldsymbol{P}^{T}\right)^{T},
$$

where, $\mathbf{B}=\left[\begin{array}{lll}B_{x} & B_{y} & B_{z}\end{array}\right]^{T}$ is body force per unit mass along $x, y$ and $z$ axes respectively, $\rho=\rho(\boldsymbol{x}, t)$ is the mass density, $\dot{\mathbf{U}}$ are velocities in $x, y$ and $z$ directions which provide the Eulerian form of reference and $\boldsymbol{P}$ the Cauchy stress tensor, which is defined in current configuration. To obtain the complete expression for stress tensor, the two tensors $\boldsymbol{P}_{\boldsymbol{d}}$ and $\boldsymbol{P}_{\boldsymbol{e}}$ which correspond to the non-elastic and elastic parts respectively are added, i.e. $\boldsymbol{P}=\boldsymbol{P}_{\boldsymbol{d}}+\boldsymbol{P}_{\boldsymbol{e}}$. Substituting the expressions obtained earlier, we get,

$$
\begin{aligned}
\rho \frac{D \dot{\mathbf{U}}}{D t}= & \rho \mathbf{B}+\nabla^{T}\left(-p+\lambda K_{1}+\lambda_{1} J_{1}+\lambda_{4} J_{2}\right)+\left[\nabla \cdot \left(2 \mu \boldsymbol{E}^{*}-3 \lambda_{1} \boldsymbol{A}\right.\right. \\
& \left.\left.-3 \lambda_{4} \operatorname{Adj}(\boldsymbol{A})\right)\right]^{T} .
\end{aligned}
$$

In the above equation of motion, $\lambda_{1}$ is similar to the classical fluid viscosity and $\lambda_{4}$ is an unknown nonlinear viscosity coefficient representing additional resistance to advection in solids. There is a new state variable called pressure, $p$, which is only a computational intermediary and agrees with classical fluid pressure when elastic resistance is not there. This variable is equivalent to mean stress in solids (Zienkiewicz \& Godbole, 1975). There are four dependent variables, the three components of the displacement vector, i.e., $u, v, w$ and a pressure quantity $p$, all of which are functions of space variables and time. Therefore to generate the additional equation required, the equation of motion has to be supplemented with the continuity equation.

The law of conservation of mass i.e. continuity equation in spatial (Eulerian) form (Malvern $1969)$ is given by,

$$
D \rho / D t+\rho(\nabla \cdot \dot{\mathbf{U}})=0
$$

where $\rho=\rho(\boldsymbol{x}, t)$ is the mass density and $\dot{\mathbf{U}}$ the velocities in $x, y$ and $z$ directions.

For convenience in the later part of the paper we will express here the mass density $\rho$ in the form of volumetric strain $v$ as,

$$
\frac{D}{D t}\left(\frac{1}{v}\right)+\frac{1}{v}(\nabla \cdot \dot{\mathbf{U}})=0
$$

The 1-D and 2-D forms of the new field equation in cartesian coordinates and in cylindrical coordinate system reduces to the same form considering the strain in the $r$ and $z$ directions. But the second unknown material constant $\lambda_{4}$ does not appear in this equation. Thus, even though it is believed that the 2-D cylindrical form in terms of $r$ and $z$ is the reduced 3-D cartesian coordinate form, it does not help us to obtain the importance of the second unknown material constant $\lambda_{4}$ which appears only in 3-D. 


\subsection{Dimensionless form of the equation of motion}

So far the equations of motion developed are complex, difficult to interpret both geometrically and physically, and to solve analytically if they are kept in the same form. These equations should be put into compact forms in order to compare their behaviour with experimental observations. Hence we carry out nondimensionalisation of the equations. This also helps (White 1994) to reduce the number and the complexity of the variables.

The dimensionless form of the 1-D equation of motion is obtained by nondimensionalising the terms as follows,

$$
\begin{aligned}
u & =\left(u / u_{0}\right) u_{0}=\bar{u} u_{0} \Rightarrow \mathrm{d} u=u_{0} \mathrm{~d} \bar{u}, \\
t & =\left(t / t_{0}\right) t_{0}=\bar{t} t_{0} \Rightarrow \mathrm{d} t=t_{0} \mathrm{~d} \bar{t}, \\
x & =\left(x / x_{0}\right) x_{0}=\bar{x} x_{0} \Rightarrow \mathrm{d} x=x_{0} \mathrm{~d} \bar{x}, \\
p & =\left(p / p_{0}\right) p_{0}=\bar{p} p_{0}, \\
\rho & =\left(\rho / \rho_{0}\right) \rho_{0}=\bar{\rho} \rho_{0}, \\
B_{x} & =\left(B_{x} / B_{x_{0}}\right) B_{x_{0}}=\bar{B}_{x} B_{x_{0}}, \\
\dot{u} & =\frac{\mathrm{d} u}{\mathrm{~d} t}=\frac{u_{0} \mathrm{~d} \bar{u}}{t_{0} \mathrm{~d} \bar{t}}=\frac{u_{0}}{t_{0}} \dot{\bar{u}}, \\
\frac{\mathrm{d} u}{\mathrm{~d} x} & =\frac{u_{0} \mathrm{~d} \bar{u}}{x_{0} \mathrm{~d} \bar{x}}, \\
\frac{\mathrm{d} \dot{u}}{\mathrm{~d} t} & =\frac{u_{0} \mathrm{~d} \dot{\bar{u}}}{t_{0} t_{0} \mathrm{~d} \bar{t}}=\frac{u_{0} \mathrm{~d} \dot{\bar{u}}}{t_{0}^{2} \mathrm{~d} \bar{t}}, \\
\frac{\mathrm{d} \dot{u}}{\mathrm{~d} x} & =\frac{u_{0} \mathrm{~d} \dot{\bar{u}}}{t_{0} x_{0} \mathrm{~d} \bar{x}},
\end{aligned}
$$

where, $u_{0}, t_{0}, x_{0} \ldots$ etc. are the known scale factors and $\bar{u}, \bar{t}, \bar{x} \ldots$ etc. are the corresponding nondimensionalised variables. Thus the 1-D form of the nondimensionalised field equation becomes,

$$
\frac{\mathrm{d} \dot{\bar{u}}}{\mathrm{~d} t}+\epsilon_{0} \dot{\bar{u}} \frac{\mathrm{d} \dot{\bar{u}}}{\mathrm{~d} \bar{x}}=\zeta \overline{B_{x}}+\bar{v} \frac{\mathrm{d}}{\mathrm{d} \bar{x}}\left[-\beta \bar{p}-2 \gamma \frac{\mathrm{d} \dot{\bar{u}}}{\mathrm{~d} \bar{x}}+\frac{1}{2} \chi\left\{2 \frac{\mathrm{d} \bar{u}}{\mathrm{~d} \bar{x}}-\epsilon_{0}\left(\frac{\mathrm{d} \bar{u}}{\mathrm{~d} \bar{x}}\right)^{2}\right\}\right],
$$

where,

$$
\begin{aligned}
\epsilon_{0} & =u_{0} / x_{0}, \\
\zeta & =B_{x_{0}} t_{0}^{2} / u_{0}, \\
\bar{v} & =1+\bar{v}_{1}=1+\left(u_{0} d \bar{u} / x_{0} d \bar{x}\right), \\
\beta & =p_{0} t_{0}^{2} /\left(\rho_{0} u_{0} x_{0}\right), \\
\gamma & =\lambda_{1} t_{0} /\left(p_{0} x_{0}^{2}\right), \\
\chi & =(\lambda+2 \mu)\left(t_{0}^{2} / \rho_{0} x_{0}^{2}\right),
\end{aligned}
$$

are the dimensionless constants. We can write $\beta$ as,

$$
\beta=\frac{p_{0} t_{0}^{2}}{\rho_{0} u_{0} x_{0}}=\frac{p_{0} t_{0}^{2}}{\rho_{0} x_{0}^{2}\left(u_{0} / x_{0}\right)} .
$$


Let us define,

$$
p_{0}=\left(u_{0} / x_{0}\right)(\lambda+2 \mu)
$$

Then we get,

$$
\begin{aligned}
\beta & =\frac{\left(u_{0} / x_{0}\right)(\lambda+2 \mu) t_{0}^{2}}{\rho_{0} x_{0}^{2}\left(u_{0} / x_{0}\right)} \\
& =\frac{(\lambda+2 \mu) t_{0}^{2}}{\rho_{0} x_{0}^{2}} \\
& =\chi
\end{aligned}
$$

Now we can write $\chi$ as,

$$
\chi=\frac{(\lambda+2 \mu) t_{0}^{2}}{\rho_{0}\left(u_{0} / x_{0}\right)^{2} u_{0}^{2}}
$$

where $\left(u_{0} / x_{0}\right)^{2}$ is the limiting strain during elasto-plastic loading say 'ultimate strain' whereas, $u_{0} / t_{0}=\dot{u}_{0}$ is the rate of deformation, i.e. the velocity at which load is applied. Further we can see that,

$$
(\lambda+2 \mu) / \rho_{0} \propto E / \rho=c^{2},
$$

where $c$ is the 1-D wave velocity in the metal, i.e.

$$
\chi=\beta=\varrho \frac{c^{2}}{\dot{u}_{0}^{2}}\left(\frac{u_{0}}{x_{0}}\right)^{2}
$$

where $\varrho$ is a constant.

From the dimensionless form we can see that $1 / \gamma$ has a form similar to that of Reynolds number Re in the case of fluids. Therefore $\lambda_{1}$ must be a material constant corresponding to the material viscosity term. Thus the 1-D equation of motion developed reduces to the simplified form

$$
\frac{\mathrm{d} \dot{\bar{u}}}{\mathrm{~d} t}+\epsilon_{0} \dot{\bar{u}} \frac{\mathrm{d}}{\mathrm{d} \overline{\bar{u}}}=\zeta \bar{B}_{x}+\bar{v} \frac{\mathrm{d}}{\mathrm{d} \bar{x}}\left[\beta\left(-\bar{p}+\frac{\mathrm{d} \bar{u}}{\mathrm{~d} \bar{x}}-\frac{1}{2} \epsilon_{0}\left(\frac{\mathrm{d} \bar{u}}{\mathrm{~d} \bar{x}}\right)^{2}\right)-2 \frac{1}{\operatorname{Re}} \frac{\mathrm{d} \dot{\bar{u}}}{\mathrm{~d} \bar{x}}\right]
$$

We can see that, during large deformation, the higher order terms of the equation play an important role.

A similar procedure is carried out for 2-D and 3-D equations of motion to get the dimensionless forms. We consider some characteristic length $\bar{l}$ and a characteristic increment in length $\bar{\Delta}$. The final form of the dimensionless 2-D equation of motion becomes,

$$
\begin{aligned}
& \left\{\begin{array}{l}
C_{1} \\
C_{2}
\end{array}\right\}=\zeta\left\{\begin{array}{l}
\bar{B}_{x} \\
\bar{B}_{y}
\end{array}\right\}+\bar{v}\left\{\begin{array}{l}
\partial / \partial \bar{x} \\
\partial / \partial \bar{y}
\end{array}\right\}\left(-\beta \bar{p}+\chi^{\prime} \bar{K}_{1}+\gamma \bar{J}_{1}\right) \\
& +\bar{v}\left[\begin{array}{cc}
\frac{\partial}{\partial \bar{x}} & \frac{\partial}{\partial \bar{y}}
\end{array}\right]\left[\chi^{\prime \prime}\left[\begin{array}{ll}
\bar{E}_{x x} & \bar{E}_{x y} \\
\bar{E}_{y x} & \bar{E}_{y y}
\end{array}\right]-3 \gamma\left[\begin{array}{cc}
\bar{a} & \bar{h} / 2 \\
\bar{h} / 2 & \bar{b}
\end{array}\right]\right],
\end{aligned}
$$


where,

$$
\begin{aligned}
\zeta & =g t_{0}^{2} / \bar{\Delta}, \quad \beta=p_{0} t_{0}^{2} / \rho_{0} \bar{l} \bar{\Delta}, \quad \gamma=\lambda_{1} t_{0} / \rho_{0} \bar{l}^{2}, \\
\chi^{\prime} & =\lambda t_{0}^{2} / \rho_{0} \bar{l}^{2}, \quad \chi^{\prime \prime}=\mu t_{0}^{2} / \rho_{0} \bar{l}^{2}, \\
\bar{v} & =1+\epsilon_{0}\left(\frac{\partial \bar{u}}{\partial \bar{x}}+\frac{\partial \bar{v}}{\partial \bar{y}}+\epsilon_{0} \frac{\partial \bar{u}}{\partial \bar{x}} \frac{\partial \bar{v}}{\partial \bar{y}}\right), \\
C_{1} & =\frac{\partial \dot{\bar{u}}}{\partial \bar{t}}+\epsilon_{0}\left(\dot{\bar{u}} \frac{\partial \overline{\bar{u}}}{\partial \bar{x}}+\dot{\bar{v}} \frac{\partial \dot{\bar{u}}}{\partial \bar{y}}\right), \\
C_{2} & =\frac{\partial \dot{\bar{v}}}{\partial \bar{t}}+\epsilon_{0}\left(\dot{\bar{u}} \frac{\partial \dot{\bar{v}}}{\partial \bar{x}}+\dot{\bar{v}} \frac{\partial \dot{\bar{v}}}{\partial \bar{y}}\right), \\
\bar{K}_{1} & =\bar{K}_{x x}+\bar{K}_{y y}, \\
\bar{K}_{x x} & =\bar{E}_{x x}=\frac{\partial \bar{u}}{\partial \bar{x}}-\frac{\epsilon_{0}}{2}\left(\left(\frac{\partial \bar{u}}{\partial \bar{x}}\right)^{2}+\left(\frac{\partial \bar{v}}{\partial \bar{x}}\right)^{2}\right), \\
\bar{K}_{y y} & =\bar{E}_{y y}=\frac{\partial \bar{v}}{\partial \bar{y}}-\frac{\epsilon_{0}}{2}\left(\left(\frac{\partial \bar{u}}{\partial \bar{y}}\right)^{2}+\left(\frac{\partial \bar{v}}{\partial \bar{y}}\right)^{2}\right), \\
\bar{E}_{x y} & =\frac{\partial \bar{u}}{\partial \bar{y}}+\frac{\partial \bar{v}}{\partial \bar{x}}-\epsilon_{0}\left(\frac{\partial \bar{u}}{\partial \bar{x}} \frac{\partial \bar{u}}{\partial \bar{y}}+\frac{\partial \bar{v}}{\partial \bar{x}} \frac{\partial \bar{v}}{\partial \bar{y}}\right)^{2}, \\
\bar{a} & =\partial \dot{\bar{u}} / \partial \bar{x}, \quad \bar{b}=\partial \dot{\bar{v}} / \partial \bar{y}, \quad \bar{h}=\partial \dot{\bar{v}} / \partial \bar{x}+\partial \dot{\bar{u}} / \partial \bar{y} .
\end{aligned}
$$

The dimensionless form of the 3 -D equation becomes,

$$
\begin{aligned}
\partial \dot{\overline{\mathbf{U}}} / \partial t+\epsilon_{0} \dot{\overline{\mathbf{U}}} \cdot \bar{\nabla} \dot{\overline{\mathbf{U}}}= & \zeta \overline{\mathbf{B}}+\bar{v} \bar{\nabla}^{T}\left(-\beta \bar{p}+\chi^{\prime} \bar{K}_{1}+\gamma \bar{J}_{1}+\eta \epsilon_{0} \bar{J}_{2}\right) \\
& +\bar{v} \nabla \cdot\left[\chi^{\prime \prime} \overline{\mathbf{E}}-3 \gamma \overline{\boldsymbol{A}}-3 \eta \epsilon_{0} \operatorname{Adj}(\overline{\boldsymbol{A}})\right],
\end{aligned}
$$

where,

$$
\begin{aligned}
\bar{K} & =\bar{K}_{x x}+\bar{K}_{y y}+\bar{K}_{z z}, \\
\bar{K}_{x x} & =\bar{E}_{x x}=\frac{\partial \bar{u}}{\partial \bar{x}}-\frac{\epsilon_{0}}{2}\left(\left(\frac{\partial \bar{u}}{\partial \bar{x}}\right)^{2}+\left(\frac{\partial \bar{v}}{\partial \bar{x}}\right)^{2}+\left(\frac{\partial \bar{w}}{\partial \bar{x}}\right)^{2}\right), \\
\bar{K}_{y y} & =\bar{E}_{y y}=\frac{\partial \bar{v}}{\partial \bar{y}}-\frac{\epsilon_{0}}{2}\left(\left(\frac{\partial \bar{u}}{\partial \bar{y}}\right)^{2}+\left(\frac{\partial \bar{v}}{\partial \bar{y}}\right)^{2}+\left(\frac{\partial \bar{w}}{\partial \bar{y}}\right)^{2}\right), \\
\bar{K}_{z z} & =\bar{E}_{z z}=\frac{\partial \bar{w}}{\partial \bar{z}}-\frac{\epsilon_{0}}{2}\left(\left(\frac{\partial \bar{u}}{\partial \bar{z}}\right)^{2}+\left(\frac{\partial \bar{v}}{\partial \bar{z}}\right)^{2}+\left(\frac{\partial \bar{w}}{\partial \bar{z}}\right)^{2}\right), \\
\bar{E}_{x y} & =\frac{\partial \bar{u}}{\partial \bar{y}}+\frac{\partial \bar{v}}{\partial \bar{x}}-\epsilon_{0}\left(\frac{\partial \bar{u}}{\partial \bar{x}} \frac{\partial \bar{u}}{\partial \bar{y}}+\frac{\partial \bar{v}}{\partial \bar{x}} \frac{\partial \bar{v}}{\partial \bar{y}}+\frac{\partial \bar{w}}{\partial \bar{x}} \frac{\partial \bar{w}}{\partial \bar{y}}\right), \\
\bar{E}_{y z} & =\frac{\partial \bar{v}}{\partial \bar{z}}+\frac{\partial \bar{w}}{\partial \bar{y}}-\epsilon_{0}\left(\frac{\partial \bar{u}}{\partial \bar{y}} \frac{\partial \bar{u}}{\partial \bar{z}}+\frac{\partial \bar{v}}{\partial \bar{y}} \frac{\partial \bar{v}}{\partial \bar{z}}+\frac{\partial \bar{w}}{\partial \bar{y}} \frac{\partial \bar{w}}{\partial \bar{z}}\right), \\
\bar{E}_{z x} & =\frac{\partial \bar{w}}{\partial \bar{x}}+\frac{\partial \bar{u}}{\partial \bar{z}}-\epsilon_{0}\left(\frac{\partial \bar{u}}{\partial \bar{z}} \frac{\partial \bar{u}}{\partial \bar{x}}+\frac{\partial \bar{v}}{\partial \bar{z}} \frac{\partial \bar{v}}{\partial \bar{x}}+\frac{\partial \bar{w}}{\partial \bar{z}} \frac{\partial \bar{w}}{\partial \bar{x}}\right), \\
\bar{a} & =\partial \dot{\bar{u}} / \partial \bar{x}, \quad \bar{b}=\partial \dot{\bar{v}} / \partial \bar{y}, \quad \bar{c}=\partial \dot{\bar{w}} / \partial \bar{z},
\end{aligned}
$$




$$
\begin{aligned}
\bar{f} & =\frac{\partial \dot{\bar{w}}}{\partial \bar{y}}+\frac{\partial \dot{\bar{v}}}{\partial \bar{z}}, \quad \bar{g}=\frac{\partial \dot{\bar{u}}}{\partial \bar{z}}+\frac{\partial \dot{\bar{w}}}{\partial \bar{x}}, \quad \bar{h}=\frac{\partial \dot{\bar{v}}}{\partial \bar{x}}+\frac{\partial \dot{\bar{u}}}{\partial \bar{y}}, \\
\bar{J}_{1} & =\bar{a}+\bar{b}+\bar{c}, \\
\bar{J}_{2} & =\bar{a} \bar{b}+\bar{b} \bar{c}+\bar{c} \bar{a}-\frac{1}{4}\left(\bar{f}^{2}+\bar{g}^{2}+\bar{h}^{2}\right),
\end{aligned}
$$

$\eta$ is the additional dimensional constant due to the unknown material constant $\lambda_{4}$ which appears only in 3-D,

$$
\eta=\lambda_{4} / \rho_{0} \vec{l}^{2}
$$

Similarly, we can write the dimensionless form of the continuity equation as,

$$
\begin{aligned}
0= & \frac{\partial}{\partial \bar{t}}\left(\frac{1}{\bar{v}}\right)+\epsilon_{0}\left(\dot{\bar{u}} \frac{\partial}{\partial \bar{x}}\left(\frac{1}{\bar{v}}\right)+\dot{\bar{v}} \frac{\partial}{\partial \bar{y}}\left(\frac{1}{\bar{v}}\right)+\dot{\bar{w}} \frac{\partial}{\partial \bar{z}}\left(\frac{1}{\bar{v}}\right)\right) \\
& +\frac{\epsilon_{0}}{\bar{v}}\left(\frac{\partial \dot{\bar{u}}}{\partial \bar{x}}+\frac{\partial \dot{\bar{v}}}{\partial \bar{y}}+\frac{\partial \dot{\bar{w}}}{\partial \bar{z}}\right) .
\end{aligned}
$$

\section{Model representation and solutions}

The governing differential equations developed in the last section represent the elasto-plastic deformation considering the effect of strain and strain rate. These equations consists of four material constants, viz. $\lambda$ and $\mu$ which are Lame's constants, $\lambda_{1}$ which appears as a material viscosity term, and $\lambda_{4}$ which is an unknown material constant. The new material constant $\lambda_{4}$ is associated with the second-order term in the governing differential equation and from dimensionless analysis it shown that it has the dimension of $\mathrm{kg} / \mathrm{m}$ with dimensionless constant $\eta$. In the literature there is no mention of such a constant and any corresponding data. In this section we look into the various material phenomena which are observed during deformation of materials under loading. Since we do not have any data on $\eta$, we concentrate on the remaining resistance terms and investigate load deflection behaviour of simple models. Linear elastic resistance can be modelled with springs and nonlinear strain displacement behaviour can also be modelled by considering nonlinear incremental analysis. Dampers do represent viscous dissipation and therefore we begin by considering solids represented by grillages of springs and dampers and use of incremental loading. Our main focus is on mild steel of which inclusions and other nonhomogeneities are an integral part.

In the newly developed governing equations we see that for the usual tension test we can neglect acceleration terms and body force terms as they are very low while compared to the applied load. Then the equation is governed by the gradients of strain and strain rate. The strain rate terms appear in the form of viscous resistance which is synonymous to material damping. To represent and to interpret the complex phenomenon occurring inside the material, we treat the material composed of a very large number of particles interconnected by springs (Timoshenko \& Goodier 1970). We treat these hypothetical spring elements in parallel with a damper (figure 2) to represent the effect of momentum transfer between the particles.

Each of these combinations of spring and damper, arranged in the form of a cubical structure with diagonal and body diagonal elements as shown in figure 2, represent in an approximate way many aspects of the resistance of a material to deformation and its directional behaviour under load. These cubes are arranged side by side (figure 3 ) to represent the complete tension 


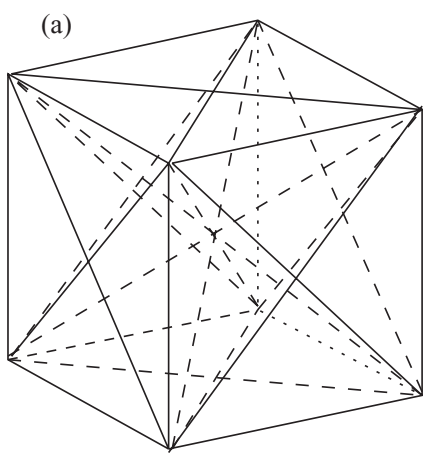

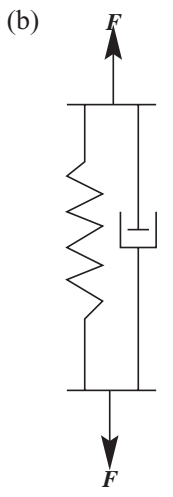

Figure 2. Spring and damper model. (a) A complete cube with elements. (b) Each element in a cube.

test specimen in length, width and depth. This whole specimen can be treated as a homogeneous material.

To start the computation, we consider a standard specimen size for the tension test prescribed in ASTM (1985) as shown in figure 4. we apply simple tension on this test piece considering only the gauge length.

However but the results of the analysis given in figure 5 show that the model does not exhibit the expected behaviour. It shows stiffening rather than the softening behaviour observed in practice, i.e. in the experimentally observed stress-strain curve for the tension test. In one sense this is expected as we use a spring element which has the characteristic behaviour of stiffening during large load.

Thus the strain and strain rate terms appearing in the governing differential equation are not enough to catch the complete material behaviour. There should be some other factor apart from the governing differential equation causes large deformation under high load. Hence we look into the effect of inhomogeneities present in the materials. In the literature we can see
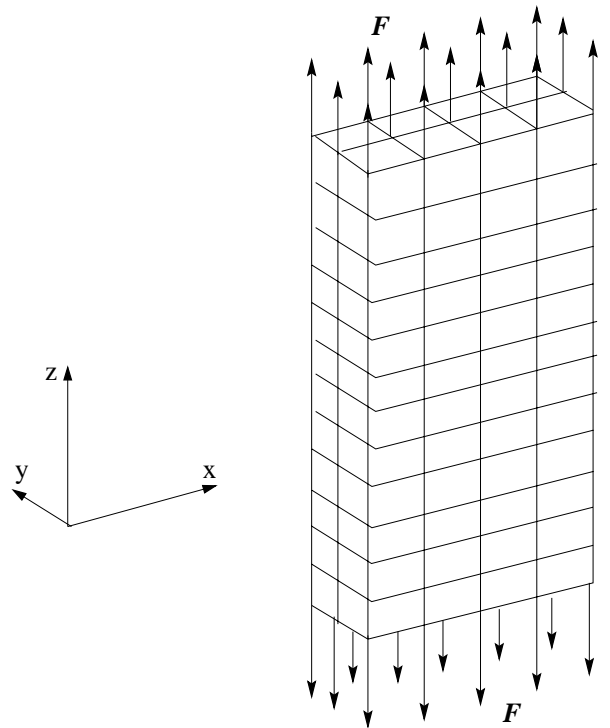

Figure 3. Tension test model. 


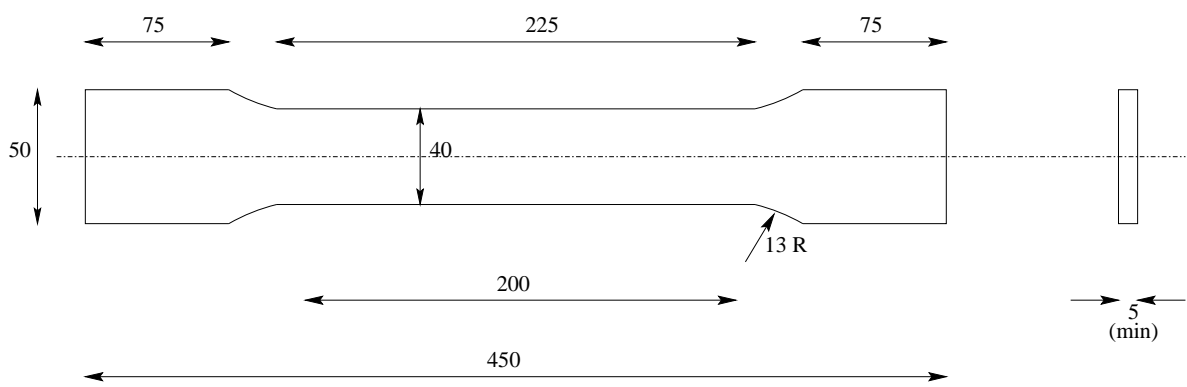

PLATE TYPE

All dimensions are in $\mathrm{mm}$

Figure 4. The standard specimen.

that much theoretical and experimental work has been carried over on these aspect leading to the microscopic theory of plasticity, which attributes the plastic deformation to microscopic inhomogeneities present in the material. Therefore we try to model the microscopic inhomogeneities as explained in the next section.

\subsection{Effect of microscopic inhomogeneities}

From the literature we can see that microscopic discontinuities in the material due to inhomogeneities result in nonuniform stress distribution in their vicinity. Stresses in these regions are higher than the average stress in the material. Values of stress concentration depend upon the size, shape and orientation of the inhomogeneity. For example, a very narrow inclusion (elongated ellipsoid) normal to the direction of force results in very high stress concentration as compared to one within the direction of force.

The inhomogeneities in our model are created by changing stiffness values of some randomly selected springs to very low values as compared to the stiffness of other springs. Due to sudden changes in stiffness values, these springs act like soft inclusions in the metal matrix.

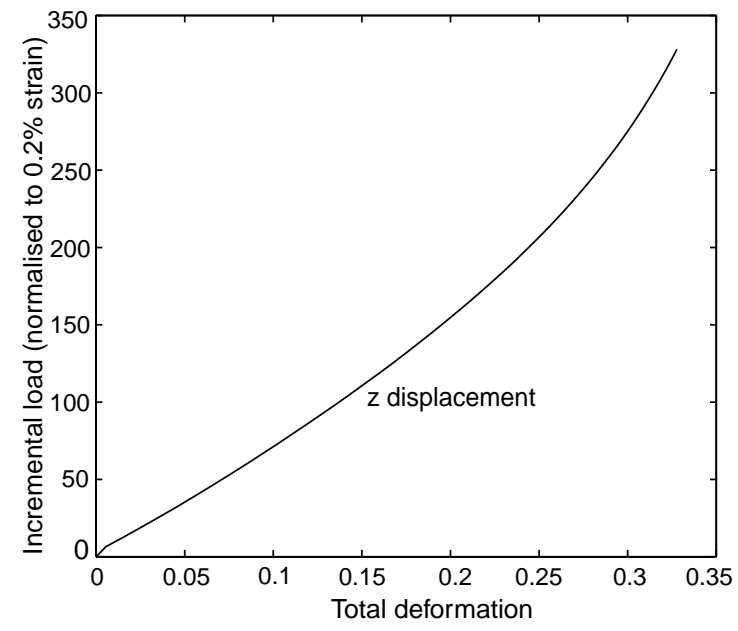

Figure 5. Effect of damping on loaddeformation behaviour. 


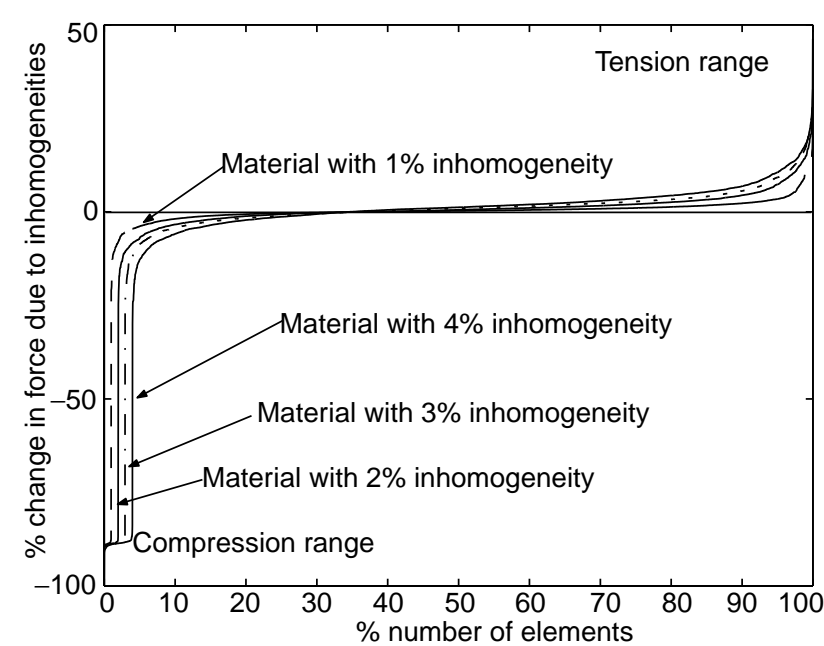

Figure 6. Comparison of material containing varying percentage of inhomogeneities.

We carry out some investigations on the orientation of "inhomogeneous" springs in the specimen to study favourable and unfavourable conditions for large deformation in the material due to different orientations of inhomogeneities. We observe maximum deformation in the case where spring elements representing nonhomogeneities are arranged in the direction of force rather than in other orientations. However actual inhomogeneities in the material are scattered in random directions. To represent random orientations, we develop random choice of elements using uniformly distributed random numbers and assign very low stiffness values to those spring elements. Typically mild steel consists of about $0.9 \%$ inhomogeneities by weight. We calculate the volumetric percentage of the inhomogeneity by knowing the densities of pure base material (ferrous) and steel, which varies from $1.2 \%$ to $2.4 \%$. Keeping in mind the volumetric percentage of inclusions, we vary the quantity of inclusion from $1 \%$ to $4 \%$ in our model by generating uniformly distributed random numbers. Comparison between percentage of inclusion and strain is tabulated in table 1. From the table we can observe that though the percentage of inhomogeneity increases, there is no great increase in the total deformation of the material as expected.

Figure 6 shows the effect of various percentages of inhomogeneity leading to large local stresses. In figure 7 we try to separate out springs representing inhomogeneities from those representing homogeneous material. Points very close to the $x$-axis show the number and the distribution of inhomogeneities. Points due to other springs are distributed along the $45^{\circ}$ line. It can be seen that some elements experience compressive stresses during tensile loading, which is to be expected.

Again the output of these studies does not bring out the expected behaviour of material (mild steel) under high load as we do not observe any softening or yield behaviour in the load

Table 1. Effect of extent of inhomogeneity on strain.

\begin{tabular}{lccccc}
\hline Extent of inhomogeneity (\%) & $0^{*}$ & 1 & 2 & 3 & 4 \\
\hline Strain & 0.0798 & 0.08 & 0.0805 & 0.0815 & 0.8115 \\
\hline
\end{tabular}

*Homogeneous material 

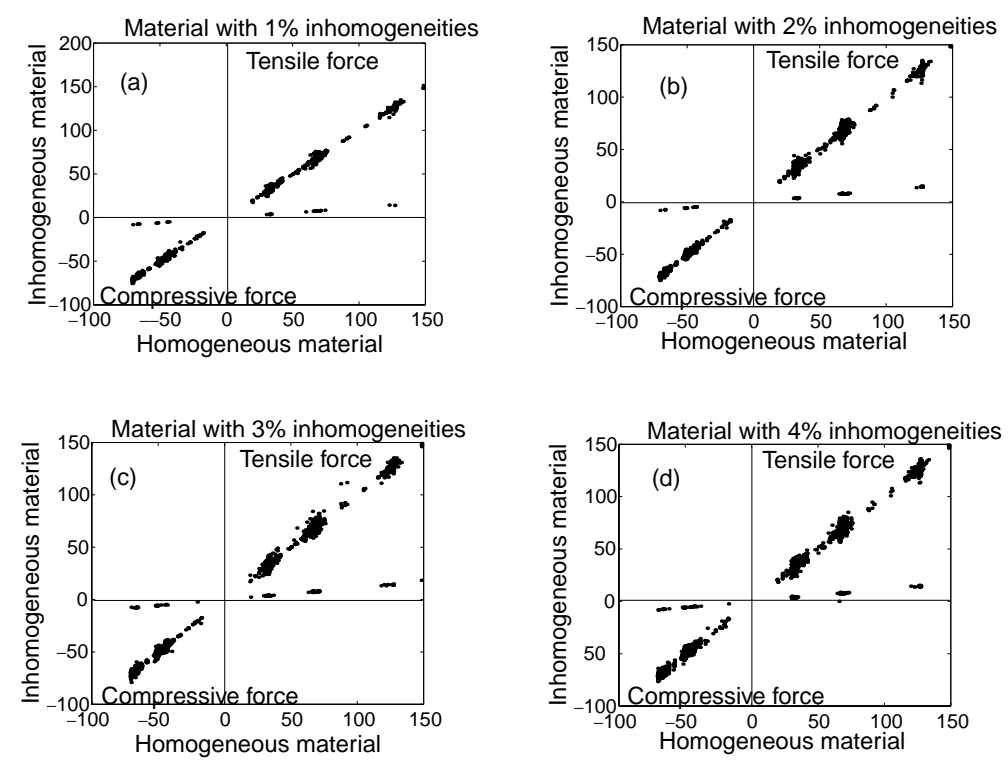

Figure 7. Distribution of spring forces for varying percentage of inhomogeneities.

verses displacement graphs. And the results are not much improved as compared to the earlier case. Thus inhomogeneities as a whole is not enough to describe material behaviour. Then the question is, what governs the observed material behaviour, i.e. yield under high load? Is it material instability?

\subsection{Effect of material instability}

The foundation of the theory of stability was laid by Euler more than two centuries ago for the case of compressive loading. But the concept of material instability remained unnoticed for a long time during the development of the elasto-plastic theory. Based upon Euler's theory and using some new concepts, Panovko \& Gubanova (1965) discuss some of the material instabilities in tensile loading which are commonly observed.

3.2a Material nonlinearity: As we have seen, material damping and nonlinear straindisplacement relations do not cause yielding or failure but only stiffen the material at high loads. The addition of inhomogeneities in our model does not cause yielding either. The experimental observations show that at higher loads materials entering into the plastic zone start showing softening behaviour and eventually get broken at some point on further application of load. Does this phenomenon occur due to loss of material stability under high load? We look into material instability and obtain a relation between the applied load and the observable strain for a simple bar considering equilibrium in the deformed configuration, thus being consistent with our field equation derived earlier. Consider a bar with cross-sectional area $A$, diameter $d$ and length $l$ fixed at one end and apply a uniform tensile load $F$ at the other end as shown in figure 8 .

Diameter after deformation is given by,

$$
d_{\varepsilon}=d(1-v \varepsilon),
$$




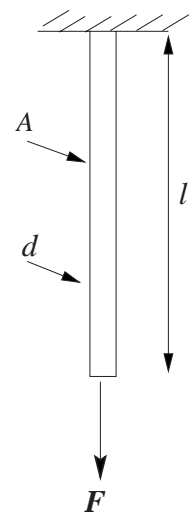

Figure 8. Bar under uniaxial tensile load.

where,

$$
\begin{aligned}
& \varepsilon=\text { equilibrium strain, } \\
& \nu=\text { Poisson's ratio, }
\end{aligned}
$$

and the cross-sectional area at equilibrium is,

$$
A_{\varepsilon}=(\pi / 4) d^{2}(1-v \varepsilon)^{2} .
$$

This cross-sectional area is the true or the equilibrium cross-sectional area due to consideration of the transverse deformation. The consideration of true cross-sectional area becomes important during large deformation and is equivalent to the use of the Eulerian co-ordinates. The stress acting on the body is,

$$
\sigma_{\varepsilon}=F / A_{\varepsilon},
$$

i.e.

$$
\begin{aligned}
E \cdot \varepsilon & =4 F /\left[\pi d^{2}(1-\nu \varepsilon)^{2}\right], \\
\Rightarrow \quad \varepsilon(1-\nu \varepsilon)^{2} & =4 F / \pi d^{2} E, \\
\Rightarrow \quad \varepsilon_{0} & =\varepsilon(1-\nu \varepsilon)^{2}
\end{aligned}
$$

where, $E$ is Young's modulus and $\varepsilon_{0}$ is $4 F /\left(\pi E d^{2}\right)$ which is the equivalent linear strain corresponding to $F$.

The behaviour of $\varepsilon_{0}$ verses $\varepsilon$ is plotted in figure 9. The maximum and minimum values of $\varepsilon_{0}$ and $\varepsilon$ can be determined by differentiating (20) with $\varepsilon$ and setting the derivative to zero, i.e.

$$
\begin{aligned}
\partial \varepsilon_{0} / \partial \varepsilon & =(1-\nu \varepsilon)^{2}-2 \nu \varepsilon(1-\nu \varepsilon) \\
& =(1-\nu \varepsilon)(1-3 \nu \varepsilon)
\end{aligned}
$$

Therefore

$$
\partial \varepsilon_{0} / \partial \varepsilon=0, \quad \text { at } \quad \varepsilon_{1}=1 / 3 v \quad \text { and } \quad \varepsilon_{2}=1 / \nu .
$$

The corresponding maximum and minimum values of $\varepsilon_{0}$ can be found by substituting back values of $\varepsilon_{1,2}$ in (20). Thus, the maximum value of $\varepsilon_{0}$ is $4 /(27 v)$ and the minimum is 0 . Let us denote the maximum value of $\varepsilon_{0}$ by $\varepsilon_{0}^{*}$ and the corresponding value of $\varepsilon$ by $\varepsilon^{*}$. 


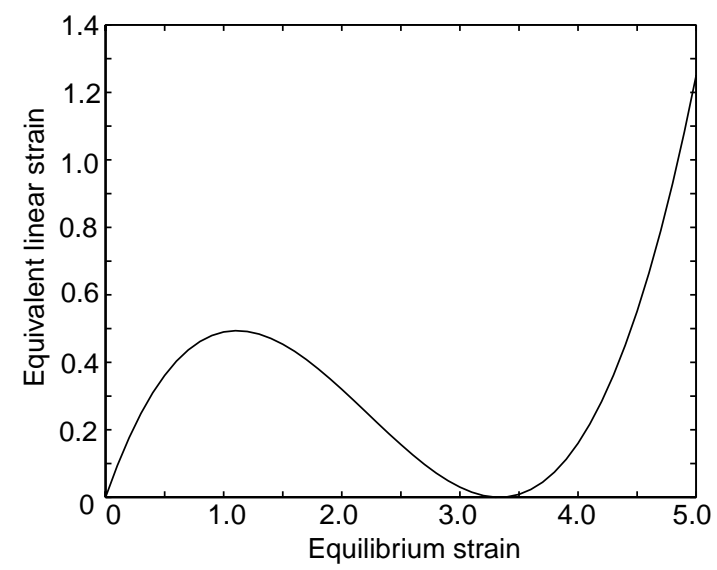

Figure 9. Material behaviour due to tension instability.

The above equation (20) is dimensionless and the material constant $v$ is the only variable, and hence we can make a general statement that for any given homogeneous material, the equivalent linear strain $\varepsilon_{0}$ can not exceed the value of $4 /(27 v)$. This is the maximum load that a body in tension can withstand. Beyond this point, materials are unstable. Eventually, material starts unloading until it breaks down. During this period, the deformation is likely to confine itself to only the neck or a weak region and the remainder of the specimen would recover elastically under decreasing load until fracture appears.

A similar graph can be obtained for the applied tension load $F$ versus the elongation at equilibrium, $l$, by substituting the values for Young's modulus $E$ and the specimen diameter $d$ in (19). It gives a maximum load value $F^{*}$ for the corresponding value of $l^{*}$ (in terms of length scale) as shown in figure 10. We can call these two quantities the critical force and the critical elongation respectively. It is possible to have the rod in an equilibrium state only up to the value of $F^{*}$ of force. Beyond this point, the equilibrium state is impossible for any higher values of the force $F$. In other words, for all practical purposes, at $F=F^{*}$ the length of the rod begins to increase with decrease in value of applied force which is shown by the downward curve in figure 10, i.e. from $\varepsilon=1 /(3 v)$ to $\varepsilon=1 / \nu$. This range corresponds to the non-equilibrium state of material. At the point when $\varepsilon=1 / v$ the material breaks i.e., the area of the rod goes to zero which is quite obvious from figures 9 and 10. This behaviour is

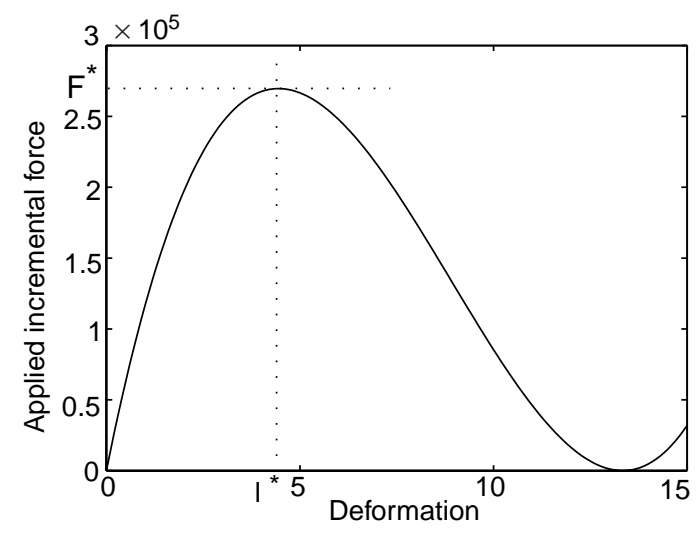

Figure 10. Material behaviour due to tension instability (force vs deformation). 
similar to the observation made by Panovko \& Gubanova (1965) in the case of loss of stability of a rod in tension. However any small irregularity in the diameter will make the rod break closer to $4 /(27 v)$ and also at a lower value of $\varepsilon_{0}$. For example, consider a uniform rod with a neck or a irregularity created in the bar artificially by removing some of the material at some position. Initially, the load will be carried by the entire bar. But later as the load increases, the deformation confines to the neck or irregularity zone due to the high stress concentration observed in these areas. In this case, the material fails at a lower load than the maximum possible.

Thus the material instability in tension has all the characteristics necessary to describe nonlinear elastic and plastic deformation and also material breakage. However, the model predicts this behaviour at very high strains of about $1 / v$ (i.e. approximately $330 \%$ in the case of steel with $v=0.3$ ) and therefore is not adequate to explain yielding of a steel test specimen which occurs around a strain of $0.1 \%$. So we take a re-look in the next section at the behaviour of materials with inhomogeneities in the light of the instabilities discussed in this section.

Before discussing the inhomogeneities, we first extend the instability criteria discussed for 1-D to 3-D. On lines similar to 1-D, we also develop a relation between equilibrium stresses versus equilibrium strains in 3-D to investigate material instability. This relation is quite useful to discuss instability surfaces which are similar to the classical yield surfaces. The 3-D version is discussed in detail in appendix A.

From the discussion on the 1-D bar, we can see that the material enters the instability zone once it reaches the critical equilibrium strain. At this point, the curve changes its direction (slope becomes negative) and starts descending and reaches zero at some particular value of equilibrium strain depending upon the value of $v$ i.e. at $1 /(3 v)$. Hence we can define a new yield criterion or instability criterion as the point in the load-deformation curve, where the slope of the equivalent strain versus equilibrium strain curve becomes zero. To extend the above to 3-D, we interpret the 1-D case as the one where $\mathrm{d} \sigma$ and $\mathrm{d} \epsilon$ are not correlated, i.e. different values of $\mathrm{d} \epsilon$ exist for the same value of $\mathrm{d} \sigma$ at this point. In 3-D we can write this relation as

$$
\mathrm{d} \sigma=C \mathrm{~d} \epsilon
$$

where $\mathrm{d} \sigma=\left(\mathrm{d} \sigma_{1}, \mathrm{~d} \sigma_{2}, \mathrm{~d} \sigma_{3}\right)^{T}$ is a vector of incremental principal stresses and $d \epsilon$ similarly is the vector of incremental principal strains $\left(\mathrm{d} \epsilon_{1}, \mathrm{~d} \epsilon_{2}, \mathrm{~d} \epsilon_{3}\right)^{T}$. Here $C$ is a tensor which gives one to one relation between incremental stresses and incremental strains in the equilibrium. The instability criterion now becomes $\operatorname{Det}[C]=0$, where $C$ is given by

$$
[\boldsymbol{C}]=\left[\begin{array}{lll}
\partial \sigma_{x} / \partial \epsilon_{x} & \partial \sigma_{x} / \partial \epsilon_{y} & \partial \sigma_{x} / \partial \epsilon_{z} \\
\partial \sigma_{y} / \partial \epsilon_{x} & \partial \sigma_{y} / \partial \epsilon_{y} & \partial \sigma_{y} / \partial \epsilon_{z} \\
\partial \sigma_{z} / \partial \epsilon_{x} & \partial \sigma_{z} / \partial \epsilon_{y} & \partial \sigma_{z} / \partial \epsilon_{z}
\end{array}\right]
$$

There are no unique solutions for $\mathrm{d} \epsilon$ for a given $\mathrm{d} \sigma$. The expression for each element in the above matrix $C$ is given in appendix A. Now we consider three different cases.

(a) $\epsilon_{x}=\epsilon_{x}$ and $\epsilon_{y}=\epsilon_{z}=-v \epsilon_{x}$ : This gives the 1-D analysis discussed so far. From figure 11a we see that for zero values of strain, the determinant is one. This point corresponds to the origin in figure 10 . When the material reaches the instability point, the determinant goes to zero. Thus, this is the point where the material changes its behaviour. In the next range, we see that value of the determinant is negative till the material breaks. 
(b) $\epsilon_{x}=\epsilon_{x}, \epsilon_{y}=\epsilon_{y}$ and $\epsilon_{z}=-v\left(\epsilon_{x}+\epsilon_{y}\right)$ : This is the 2-D case in principal strains. In this the determinant is a surface in equilibrium strains $\epsilon_{x}$ and $\epsilon_{y}$. The contour on the $\epsilon_{x}-\epsilon_{y}$ plane where the determinant is zero is the instability curve corresponding to the yield curve in 2-D and is shown in figure $11 \mathrm{~b}$.

(c) $\epsilon_{x}=\epsilon_{x}, \epsilon_{y}=\epsilon_{y}, \epsilon_{z}=\epsilon_{z}$ : The instability surface obtained in 3-D case is explained step by step in figures $11 \mathrm{c}$ to $\mathrm{f}$. Here we observe that when the values of the strains are small as compared to $1 /(3 v)$, the instability surface (determinant $=0$ surface) does not appear. As the strain values approach $1 /(3 v)$ material is seen entering the instability zone and we observe the development of the instability surface for which the determinant value is zero (figure 11c). Further figures show the development of the second surface as the value of the determinant goes to zero at the breakage point. The region outside of the instability surface is the instability zone.

3.2b Behaviour of various terms near an inclusion: To continue our search for a suitable model, we now discuss the stress fields around inhomogeneities. To begin with we consider inclusion/inhomogeneities as spherical in 3-D, and around this region see how the various terms appearing in the new field equation behave. With spherical voids, Qui \& Weng (1992) observed excellent agreement with exact solution under hydrostatic loading and the finite element calculations are in accord with tensile behaviour of material.

In the case of 3-D, the complete stress at any point around spherical inclusion is obtained as discussed by Timoshenko \& Goodier (1970) in a polar coordinate system.

These equations are later transferred back to cartesian coordinates using the transformation matrix and the graphs are plotted. Figures 12 to 14 show the behaviour of various terms near an inclusion. It is interesting to note that there is a sharp variation within the length of two radii of the inclusion.

In figure 12 we see that the invariants of linear and nonlinear strains which appear in the new field equations gradually change away from the cavity and later get stabilised at some fixed positive value. The same phenomenon can be observed with the first invariant of the strain rate but the second invariant of the strain rate undergoes a sharp change before it is stabilised at some negative value. On the other hand, gradients of the invariants for strain and strain rate change sharply before stabilising at zero.

Figure 13 shows a similar trend with the strains and their gradients. Figure 14 shows the behaviour of strain rates and their gradients. Here also we see that axial and shear strain rate terms show sharp variations within a length of twice the radius before stabilising at some constant values. As before, gradients of strain rate quantities show sharp variations before going to zero.

In the case of the pressure term $p$ we observe that the magnitude of pressure remains constant through the body, which means that the gradient of the pressure term is zero. A few earlier authors have observed that $p$ does not contribute to plastic deformation.

We observe that nonlinear behaviour or region of instability of material occurs around the value of strain equal to $1 / v$. But in practice, such a high value of strain is not observed during tension testing. Typically, in the case of steel, yield behaviour occurs at around a strain of 0.001 and the breaking phenomenon occurs at around 0.05 depending upon strain rate. Now, will we be able to derive this behaviour from what we learnt so far from our bar in tension?

To bring down the value of the critical load, $F^{*}$, and the corresponding critical strain during material instabilities to within the observed range, we introduce two new material variables $m$ and $n$, which correspond to force correction factor and strain correction factor respectively. Amongst the two, strain correction factor or actually strain enhancement factor is based on the earlier discussion on computed strains around a spherical cavity. We find there that up 


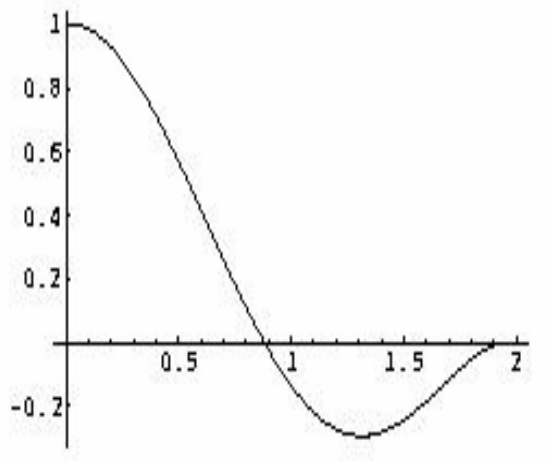

(a)

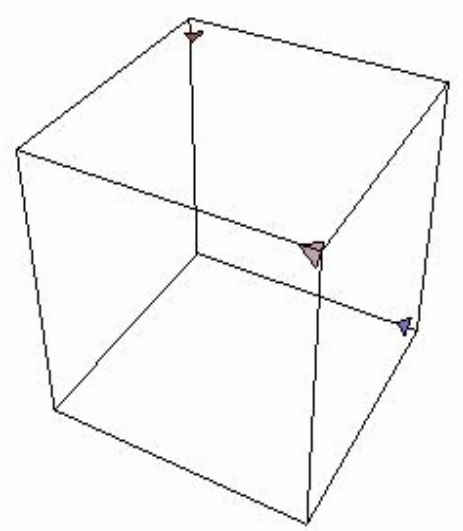

(c)

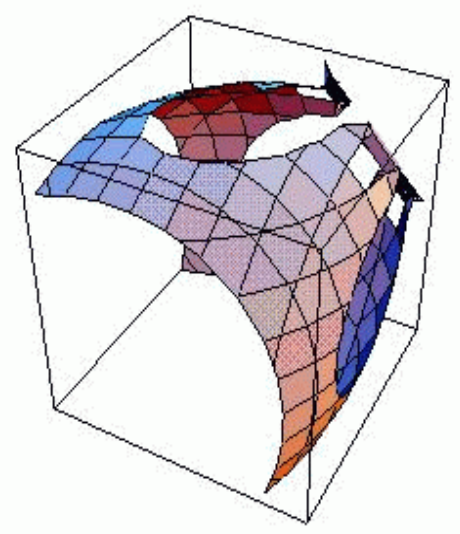

(e)

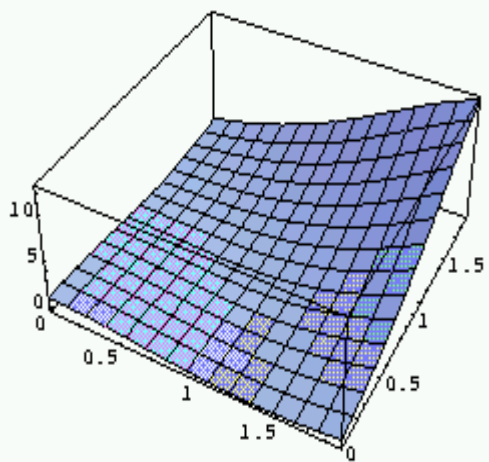

(b)

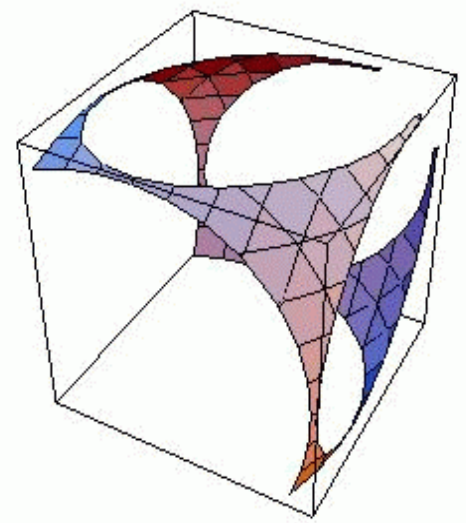

(d)

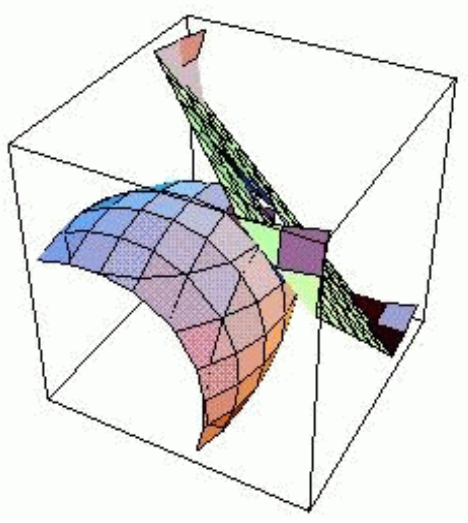

(f)

Figure 11. Development of instability surface: (a) 1-D case; (b) 2-D case; (c) 3-D case with determinant zero; (d) 3-D case with negative determinant; (e) 3-D case with determinant approaching zero; (f) 3-D case breaking point. 

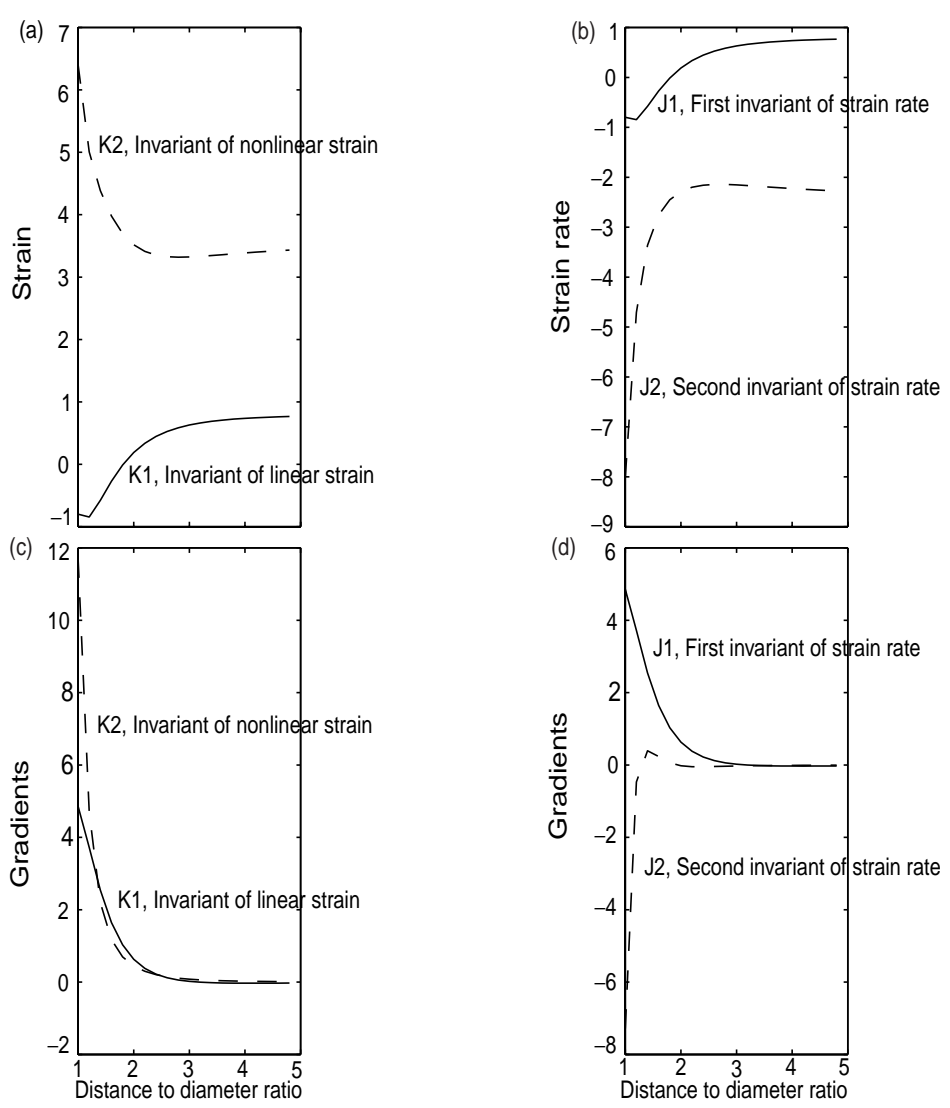

Figure 12. Behaviour of invariants near inclusion: Invariants of linear and nonlinear strain (a) and strain rate (b); Gradients of invariants of linear and nonlinear strain (c) and strain rate (d).

to $2 r$ from the cavity the average strains are larger than the nominal applied strain. This would mean that for $1 \%$ volume of nonhomogeneities in the material, about $27 \%$ of the volume has above average strain. Since the instability would see a local strain, the material behaves as if it experiences an enhanced average strain. The level of enhancement would depend on the geometry of the inhomogeneity. A value of 3 for a spherical cavity could become $a / b$ for an ellipsoidal cavity where $a$ is the major axis and $b$ is the minor axis. Thus for a flat cavity where $a / b$ would tend to $\infty$, average stress can become significantly larger than the nominal stress. This effect is represented by $n$, while the overall load-elongation behaviour in the stable range is determined by the strains corresponding to the nominal stress and strain enhancer $n$. The maximum load that can be applied depends on the section at which average stresses would peak. This section acts like the notch or groove introduced in the middle of the tension test specimen sometimes. This factor is brought in as $m$.

Figure 15 shows the behaviour of a rod which is also considered as a spring, for different values of $n$. In the graph, 'ep1' represents the behaviour of an ordinary spring while 'ep2' is that of the spring with different values of $n$. We can see that the factor $n$ governs the maximum strain at break point. An increase in value of $n$ reduces the strain-carrying capacity of the material. Figure 16 shows the behaviour as $m$ changes for a given fixed value of $n$. From the graph we see that higher values of $m$ lead to lower load-carrying capacity for a fixed value of 

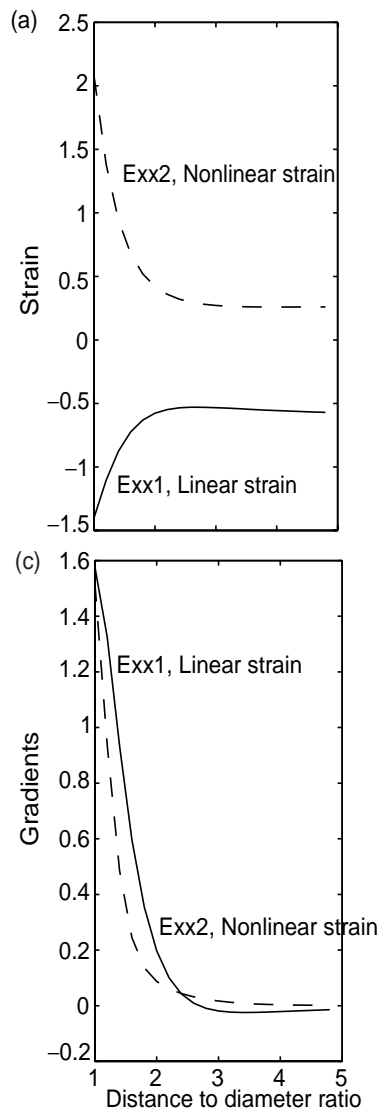
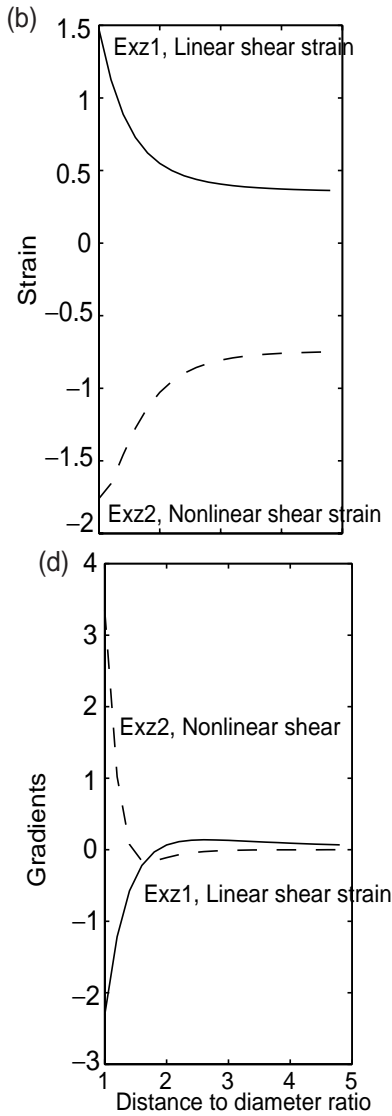

Figure 13. Behaviour of linear and nonlinear strains near inclusion: Linear and nonlinear strain (a) and shear strain (b); Gradients of linear and nonlinear strain (c) and shear strain (d).

$n$. A particular type of spring element analogous to the experimentally observed maximum load and the maximum displacement range for a given material can be generated by varying the values of $n$ and $m$. Figure 17 shows the combined effect of all these variables.

We try to match the experimentally observed phenomenon by arranging two or more springs both with and without correction factors $m$ and $n$ in some particular order. We study three different spring arrangements. In the first case, two springs are arranged in series, while they are in parallel with each other in the second case. The third case is generated by placing a spring in parallel with a combination of two springs in series. We discuss each case in detail in the following section.

\subsection{Springs in series}

Consider two springs $k_{1}$ and $k_{2}$ each of length $l_{1}$ and $l_{2}$ arranged in series with each other. Let $F$ be the applied force causing deformations of magnitudes $\Delta_{1}$ and $\Delta_{2}$ respectively as shown in figure 18.

The total deformation of the combined spring is calculated by the addition of $\Delta_{1}$ and $\Delta_{2}$. Let $n$ and $m$ be the two correction factors as discussed earlier, applied to one of the springs. 

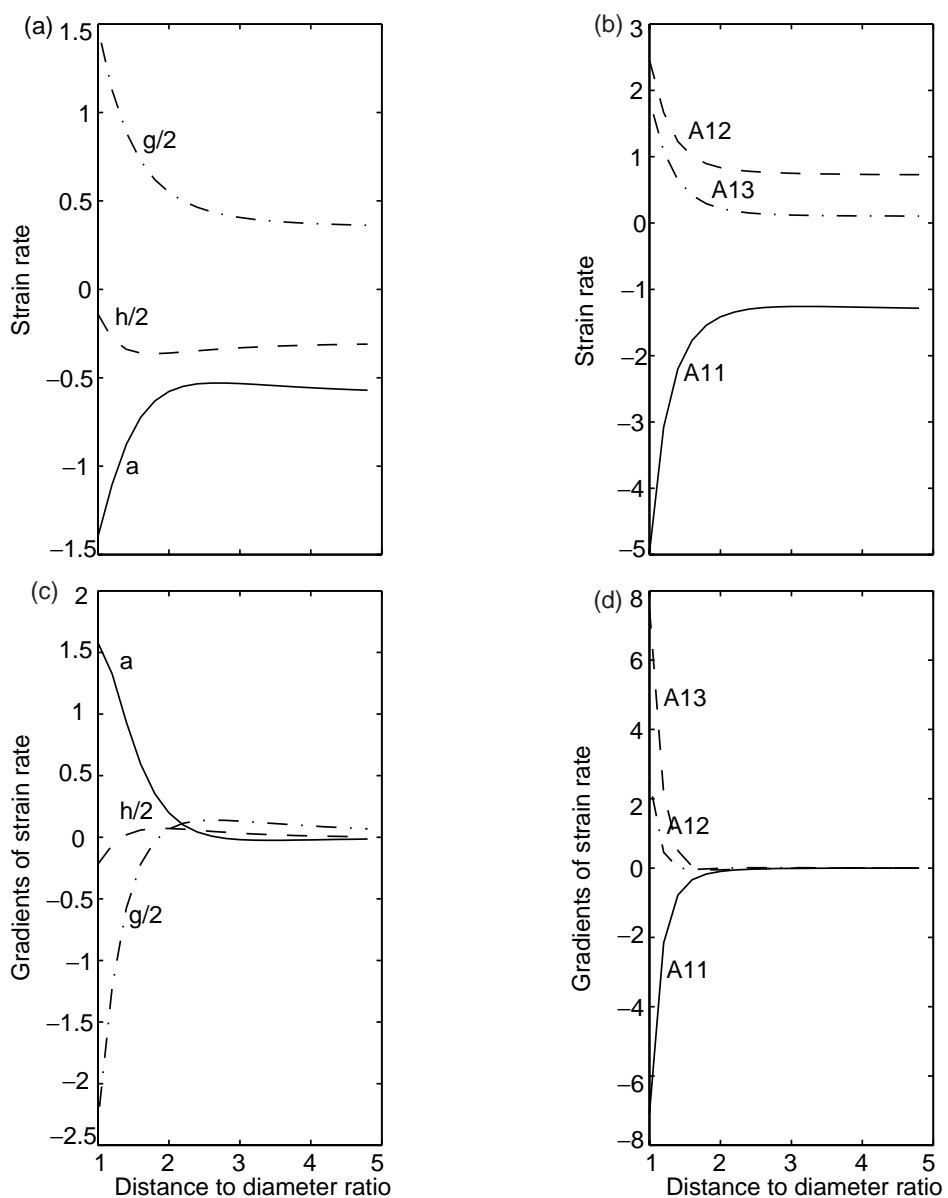

Figure 14. Behaviour of linear and nonlinear strain rates near inclusion: Linear (a) and nonlinear (b) strain rates; Gradients of linear strain rate (c) and nonlinear strain rate $(\mathbf{d})$.

The force sustained by the ordinary spring is given by,

$$
F=F_{0}\left(1-v \frac{\Delta_{1}}{l_{1}}\right)^{2} \frac{\Delta_{1}}{l_{1}},
$$

and for the other spring,

$$
F=\frac{F_{0}}{m}\left(1-n v \frac{\Delta_{2}}{l_{2}}\right)^{2} n \frac{\Delta_{2}}{l_{2}},
$$

where, $F_{0}$ is the force acting on each spring. We know that,

$$
\begin{aligned}
\Delta_{1} & =l_{1} \varepsilon_{1}, \\
\Delta_{2} & =l_{2} \varepsilon_{2}, \\
\Delta & =l_{1} \varepsilon_{1}+l_{2} \varepsilon_{2} .
\end{aligned}
$$




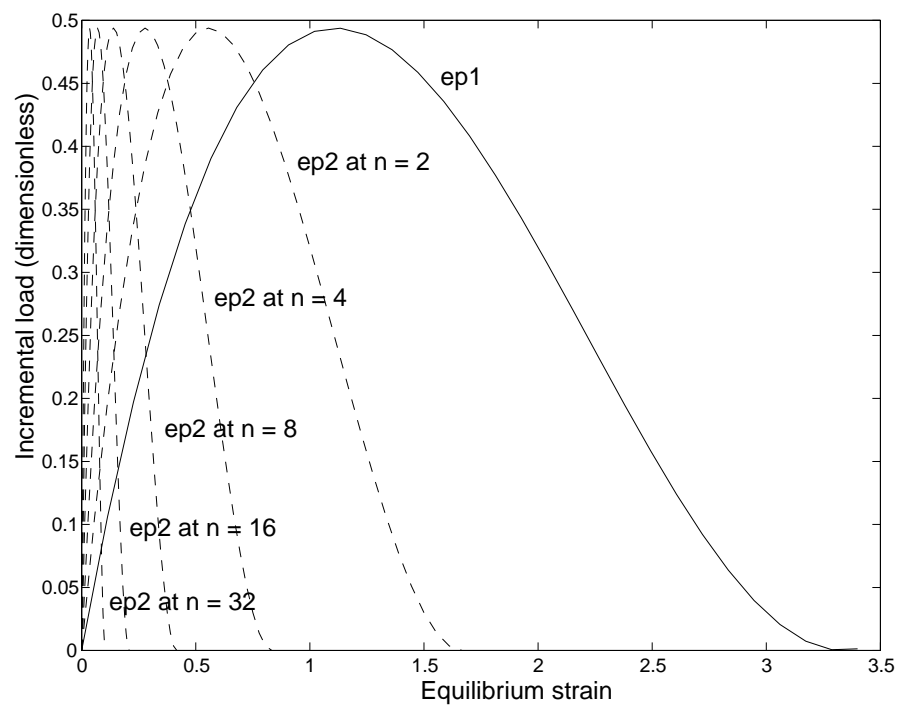

Figure 15. Effect on equilibrium strain due to change in values of $n$.

In the case of springs in series, to determine the effective strain due to the application of load, we introduce a new variable $\alpha$ such that,

$$
\begin{aligned}
& \Delta_{1}=\alpha l \varepsilon_{1} \\
& \Delta_{2}=(1-\alpha) l \varepsilon_{2}
\end{aligned}
$$

\section{Therefore}

$$
\Delta=\alpha l \varepsilon_{1}+(1-\alpha) l \varepsilon_{2}
$$

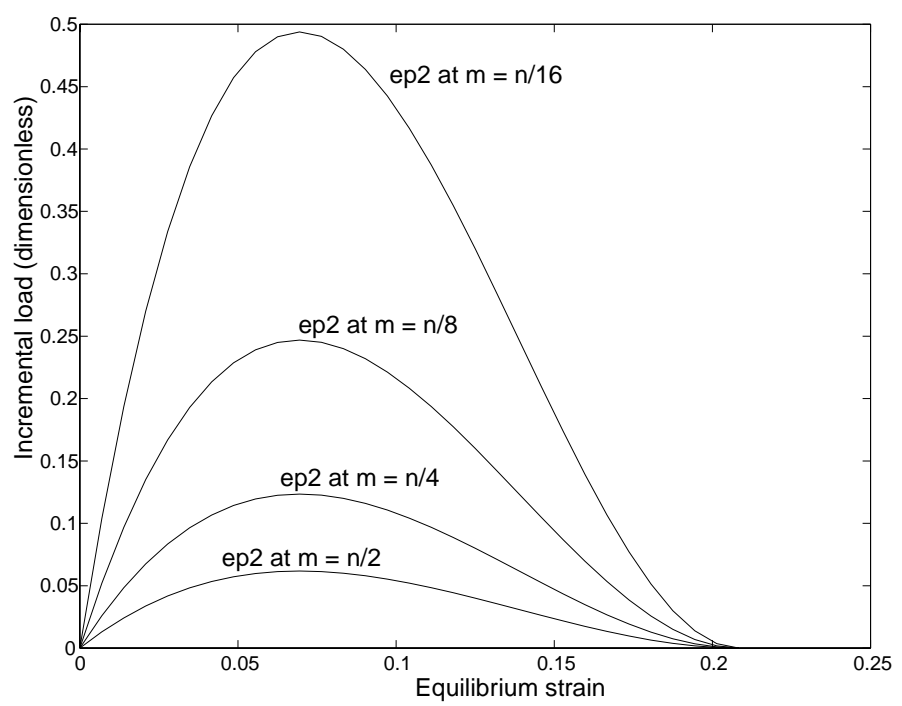

Figure 16. Effect on force due to change in values of $m$. 


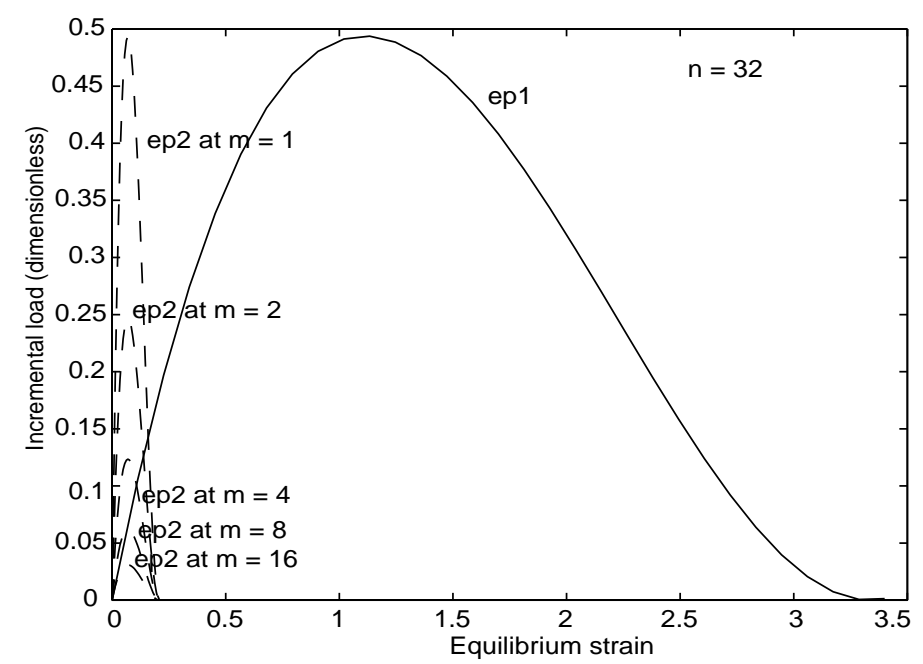

Figure 17. Combined effect on spring due to change in values of $n$ and $m$.

and

$$
\varepsilon=\Delta / l=\alpha \varepsilon_{1}+(1-\alpha) \varepsilon_{2} .
$$

The value of $\alpha$ is determined by the load-sustaining capacity of each spring. In the case of materials, we represent $\alpha$ as the effect of inhomogeneities present in the specimen.

When a set of two springs are arranged in series, the load-carrying capacity of the set is governed by the weaker spring. Figure 19 explains this behaviour. The weaker spring quickly reaches its maximum force value and then starts unloading. To be in equilibrium, the other spring also unloads such that both springs carry the same amount of force. After some time the weaker spring breaks and the other spring also gets fully unloaded. This causes some amount of residual strain in the second spring. The magnitude of residual strain depends upon the value of $\alpha$. If the value $\alpha$ is high, then the amount of residual strain is very low and in other cases it is vice versa as shown in figure 20. In the hypothetical case when the value of $\alpha$ becomes 1 , we get only one line starting from the origin as in figure 20 i.e. there are no residual strains experienced by the material. In other words, the entire material is treated as

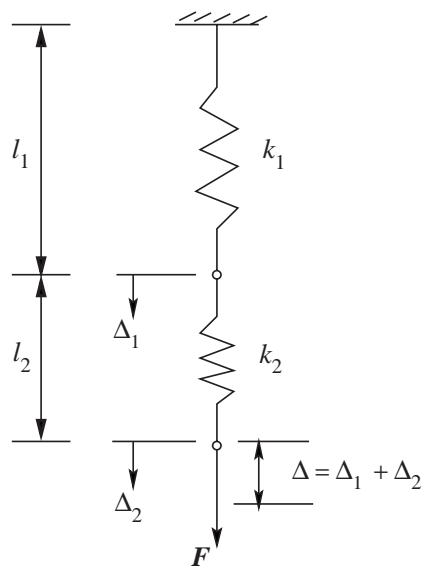

Figure 18. Two springs in series. 


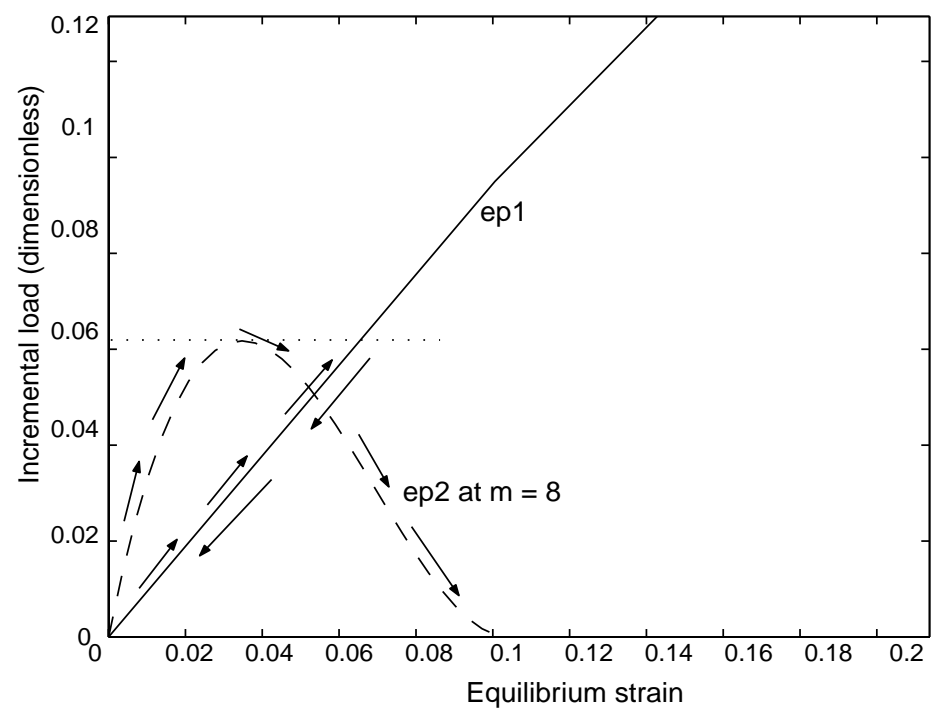

Figure 19. Effect of a weaker spring in series.

defect-free and its load-carrying capacity becomes very high as observed by Griffith (1921) in his experiments with fine glass fibres. (The subject is discussed in great detail by Timoshenko 1986).

\subsection{Springs in parallel}

Consider two springs with stiffnesses $k_{1}$ and $k_{2}$ arranged in parallel with each other. Let $\Delta$ be the deformation caused due to the application of axial load $F$ as shown in figure 21 . The

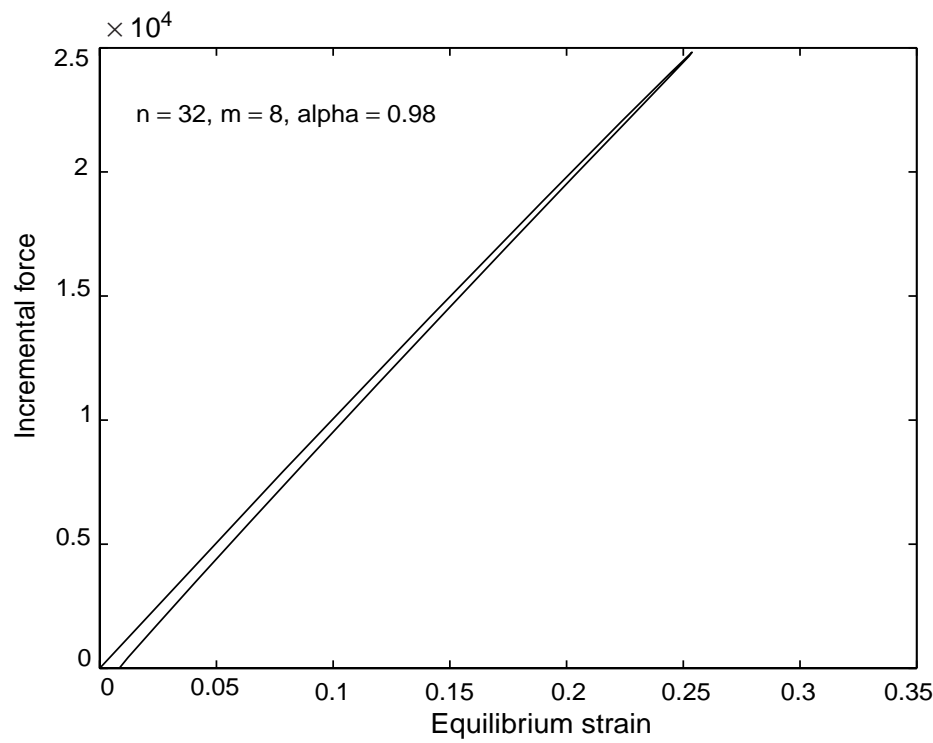

Figure 20. Effect of two different kinds of springs in series. 


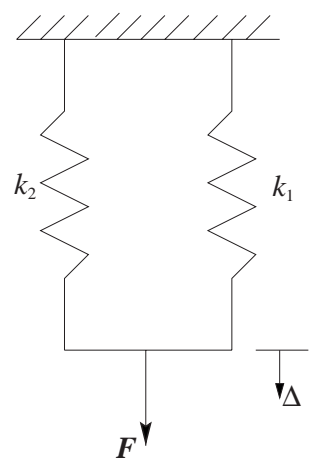

Figure 21. Two springs in parallel.

force carried by each spring is given by,

$$
\begin{aligned}
& F_{1}=F_{0}(1-v \varepsilon)^{2} \varepsilon, \\
& F_{2}=\left(F_{0} / m\right)(1-n v \varepsilon)^{2} n \varepsilon,
\end{aligned}
$$

where $F_{1}$ and $F_{2}$ are forces experienced by each spring and $F_{0}$ is the total force acting on it, $n$ and $m$ are the two new material variables which govern strain and load-carrying capacity of the springs respectively and $\varepsilon$ is the equilibrium strain acting on the spring. The total force sustained by the springs in parallel is given by

$$
F=F_{1}+F_{2} .
$$

In the case of two parallel springs, the total force $F$ is the sum of the force-carrying capacity of each spring. The spring with higher values of $n$ and $m$ is considered the weaker spring in the combination. So even if the weaker spring breaks, the load continues to be carried by the stronger spring. This effect is shown in figure 22 where the effect of different values of $m$ for a particular value of $n$ is presented. We can observe a lift in the total force-carrying capacity, i.e., the curve does not touch the zero force line even after the weaker spring is broken. This is owing to the stronger spring which is still able to carry the applied load. In general, this behaviour imitates the upper and lower yield point phenomena in the material. The upper yield point appears when the weaker spring enters the instability zone and it continues up to the lower yield point where it breaks. On increasing the load, further deformation takes place in the second spring.

\subsection{Combination of series and parallel springs}

Let $k_{1}$ and $k_{2}$ be two springs arranged in series with each other. The combination of these two springs is placed in parallel with a third spring $k_{3}$. Let $\Delta_{1}$ be the displacement due to spring $k_{1}$ and $\Delta$ be the total displacement of the combined springs due to the application of load $F$ as shown in figure 23. In this combination, one of the springs in the series is an ordinary spring and other two are modified springs.

Figure 24 describes step-by-step the observed behaviour of the combined springs. In figure $24 \mathrm{a}$ we see the individual behaviour of ordinary springs and the modified spring in series separately. Figure 24b gives the combined effect of these two springs for a given value of $\alpha$. Figure $24 \mathrm{c}$ shows the individual behaviour of the spring which is in parallel, while figure $24 \mathrm{~d}$ gives the combined effect of all three springs which is similar to the commonly observed 


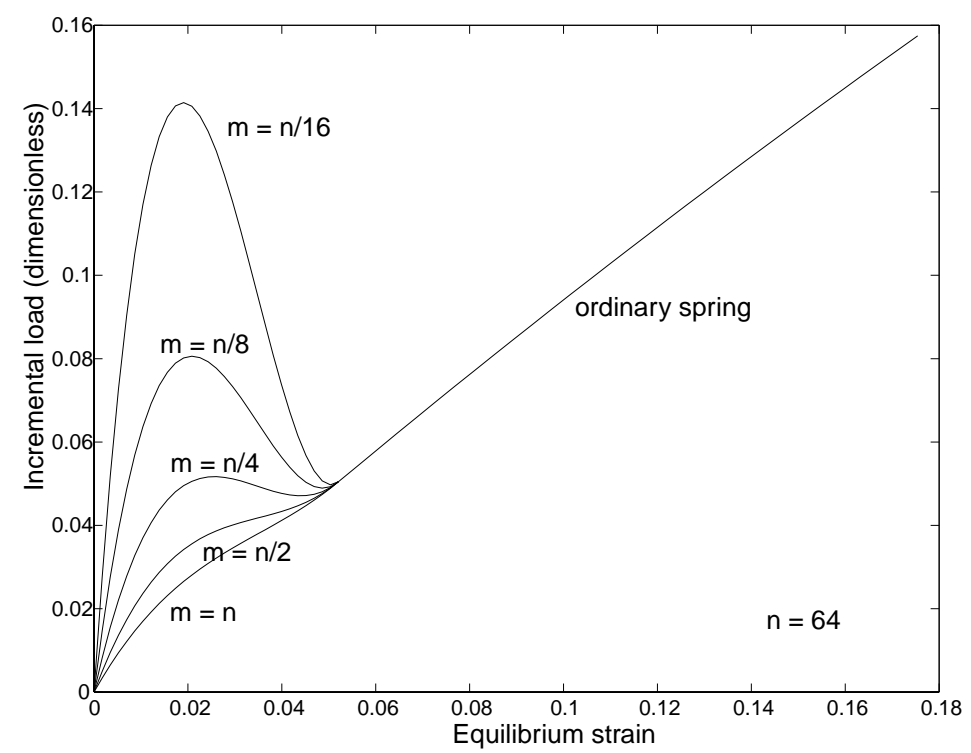

Figure 22. Combined effect of two different kinds of springs in parallel.

experimental curve for strain versus applied load. The final shape of the curve and the strain range within which it acts depend upon the values of $m, n$ and $\alpha$.

\subsection{Summary}

3.6a Field equations: Development of a new field equation and its dimensionless form in $\S 2$ and the subsequent material behaviour observed in the earlier part of this section show that there are three different kinds of displacement fields acting on a body. The first causes scaling uniform increase or decrease of density in the material. The final shape of the material remains the same but only the size varies. This effect results in a uniform stress field throughout the material. Hydrostatic tension or compression causes such deformation and we observe volumetric strain.

The second displacement field is one which changes the shape of the material after deformation without changing its density. Such deformation occurs due to the deviatoric part of the stress field. These stress fields do not cause any volumetric strain in the material and the total volume remains constant.

The third displacement field causes only rotation inside the material and does not result in any physical deformation. These displacements come from the stresses occurring on the interior and exterior boundaries of the material.

The various displacement fields mentioned above could be present simultaneously in a material. These fields are constrained by the continuity equation. More work is required to effectively use these fields to obtain solutions to the field equations.

3.6b Material inhomogeneity and instability: In this section we see that material behaviour under load can be represented by some typical arrangement of springs and dampers. Addition of damping element helps to stabilise the mathematical model under high load. The nonlinear nature of the springs observed during the case of instability tries to soften the material. Microscopic inhomogeneities cause nonuniform stress distribution in the material 


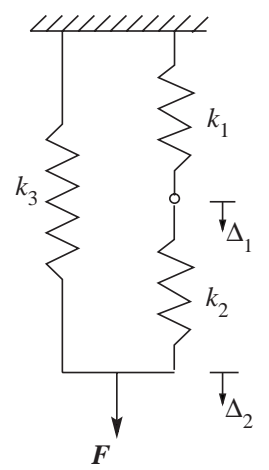

Figure 23. Two series springs in parallel with a third spring.

due to development of stress concentration in the vicinity of discontinuity or inhomogeneity. It is also observed that the orientation of inhomogeneity governs the final deformation. Normally, mild steel consists of around 0.9 wt $\%$ or 1.2 to 2.4 vol \% of inhomogeneity. The cause and effect of instability is brought out in this section. It appears that large strain deformation in the materials is due to the instabilities occurring inside the material. The representation of instability in the material using springs can be brought to the experimentally observed range by varying the values of two spring correction factors $m$ and $n$ and the different types of spring arrangements discussed. The model is also able to explain the
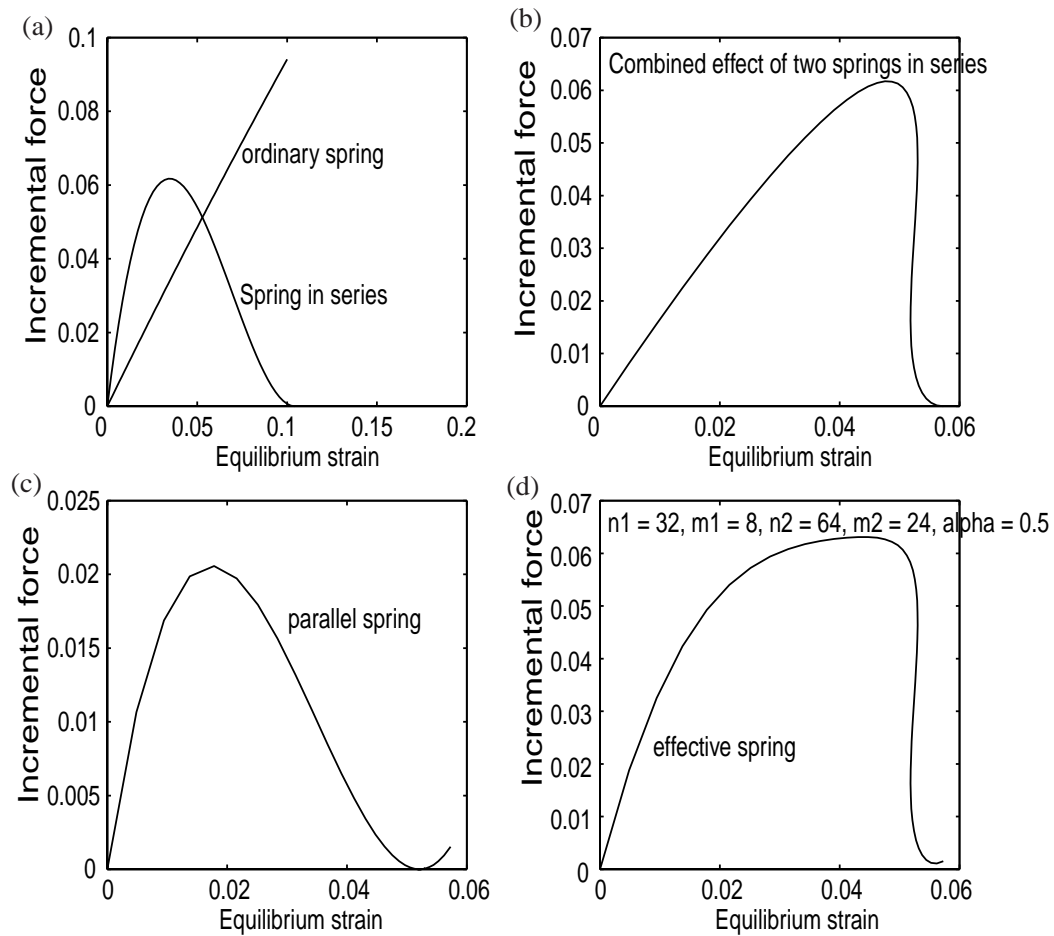

Figure 24. Step by step evolution of material behaviour under tensile load. (a) Individual behaviour of the ordinary and the modified spring in series; (b) Combined effect of ordinary and the modified springs; (c) Individual behaviour of the spring in parallel; (d) Combined effect of all three springs. 
observations from Griffith's experiments with thin wires. The practically observed material phenomena which normally occur in sheet metal-forming processes like Bauschinger effect, material anisotropy, yield point phenomenon, stress inhomogeneity, solid solution strengthening, strengthening from fine particles, fracture, residual stresses, etc. are explainable with this new model.

\section{Reformulation}

\subsection{Field equation}

The understanding of material behaviour in $\$ 3$ shows that material deformation is only due to changes in size and shape. These two factors are governed by strain and strain rate. The field equation developed in the earlier section consists of resistance due to three components: one is strain, the second is the dissipative part due to strain rate and the third is the pressure term. In the case of solids the pressure term appears as a hydrostatic stress acting on the body. From classical mechanics, it is known that deformation occurrs because of continuous action of hydrostatic and deviatoric stresses. So going back to Lamb (1945) for the original formulation and considering only small strain, we get

$$
\begin{aligned}
& p_{1}^{\prime}=-p+\lambda_{1}\left(\epsilon_{1}^{\prime}+\epsilon_{2}^{\prime}+\epsilon_{3}^{\prime}\right)+\lambda_{2} \epsilon_{1}^{\prime}, \\
& p_{2}^{\prime}=-p+\lambda_{1}\left(\epsilon_{1}^{\prime}+\epsilon_{2}^{\prime}+\epsilon_{3}^{\prime}\right)+\lambda_{2} \epsilon_{2}^{\prime}, \\
& p_{3}^{\prime}=-p+\lambda_{1}\left(\epsilon_{1}^{\prime}+\epsilon_{2}^{\prime}+\epsilon_{3}^{\prime}\right)+\lambda_{2} \epsilon_{3}^{\prime},
\end{aligned}
$$

where $\epsilon_{1}^{\prime}, \epsilon_{2}^{\prime}, \epsilon_{3}^{\prime}$ are the strains along each principal axes. Now, let us redefine the constant $\lambda_{2}$ as,

$$
p_{1}^{\prime}+p_{2}^{\prime}+p_{3}^{\prime}=\lambda_{2}\left(\epsilon_{1}^{\prime}+\epsilon_{2}^{\prime}+\epsilon_{3}^{\prime}\right) .
$$

Therefore from the definition of $p$ we get,

$$
p=\left(p_{1}^{\prime}+p_{2}^{\prime}+p_{3}^{\prime}\right) / 3=\lambda_{2}\left(\epsilon_{1}^{\prime}+\epsilon_{2}^{\prime}+\epsilon_{3}^{\prime} / 3\right)
$$

Substituting for $p$ in (28) and rearranging the terms we get,

$$
\boldsymbol{P}_{\boldsymbol{e}}^{\prime}=\lambda_{1}\left(\epsilon_{1}^{\prime}+\epsilon_{2}^{\prime}+\epsilon_{3}^{\prime}\right) \boldsymbol{I}+\lambda_{2}\left(\left[\begin{array}{ccc}
\epsilon_{1}^{\prime} & 0 & 0 \\
0 & \epsilon_{2}^{\prime} & 0 \\
0 & 0 & \epsilon_{3}^{\prime}
\end{array}\right]-\frac{\epsilon_{1}^{\prime}+\epsilon_{2}^{\prime}+\epsilon_{3}^{\prime}}{3} \boldsymbol{I}\right),
$$

which is in the form of total stress on the body expressed by the hydrostatic and deviatoric stresses. The values of $\lambda_{1}$ and $\lambda_{2}$ can be calculated from uniaxial tension and torsional tests. From the torsional test we get,

$$
\begin{aligned}
p_{1}^{\prime} & =\tau, \quad p_{2}^{\prime}=-\tau, \quad p_{3}^{\prime}=0, \\
\epsilon_{1}^{\prime} & =\tau / 2 G, \quad \epsilon_{2}^{\prime}=-\tau / 2 G, \quad \text { and } \quad \epsilon_{3}^{\prime}=0,
\end{aligned}
$$

where $\tau$ is shear stress. Substituting in (30) and solving for $\lambda_{1}$ and $\lambda_{2}$ we get,

$$
\lambda_{2}=2 G
$$


where $G$ is modulus of rigidity. From the uniaxial tension test,

$$
\begin{aligned}
& p_{1}^{\prime}=\sigma, \quad p_{2}^{\prime}=p_{3}^{\prime}=0, \\
& \epsilon_{1}^{\prime}=\sigma / E, \quad \epsilon_{2}^{\prime}=\epsilon_{3}^{\prime}=-v \sigma / E .
\end{aligned}
$$

Substituting in (30) and solving for $\lambda_{1}$ we get,

$$
\lambda_{1}=K
$$

where $K$ is bulk modulus. Thus (30) becomes the resistance due to small strains as,

$$
\boldsymbol{P}_{\boldsymbol{e}}^{\prime}=K\left(\epsilon_{1}^{\prime}+\epsilon_{2}^{\prime}+\epsilon_{3}^{\prime}\right) \boldsymbol{I}+2 G\left(\left[\begin{array}{ccc}
\epsilon_{1}^{\prime} & 0 & 0 \\
0 & \epsilon_{2}^{\prime} & 0 \\
0 & 0 & \epsilon_{3}^{\prime}
\end{array}\right]-\frac{\left(\epsilon_{1}^{\prime}+\epsilon_{2}^{\prime}+\epsilon_{3}^{\prime}\right)}{3} \boldsymbol{I}\right) .
$$

Similarly, the resistance due to rate of deformation can be determined. Consider $\dot{\epsilon}_{1}^{\prime}, \dot{\epsilon}_{2}^{\prime}, \dot{\epsilon}_{3}^{\prime}$ as the rate of strain in each direction in the principal coordinate system. Now, we can rewrite (30) for strain rate terms by substituting the strain terms $\epsilon_{1}^{\prime}, \epsilon_{2}^{\prime}, \epsilon_{3}^{\prime}$ by $\dot{\epsilon}_{1}^{\prime}, \dot{\epsilon}_{2}^{\prime}, \dot{\epsilon}_{3}^{\prime}$ with two more new constants $\lambda_{3}$ and $\lambda_{4}$. The value of new constant $\lambda_{4}$ can be found using the torsion test on a thin tube specimen.

$$
\begin{aligned}
& p_{d 1}=-\tau, \quad p_{d 2}=\tau, \quad p_{d 3}=0, \\
& \dot{\epsilon}_{1}^{\prime}=-\tau / 2 \mu, \quad \dot{\epsilon}_{2}^{\prime}=\tau / 2 \mu, \quad \dot{\epsilon}_{3}^{\prime}=0, \\
& \Rightarrow \quad \lambda_{4}=2 \mu,
\end{aligned}
$$

where $\mu$ is material viscosity term in the case of solids. The remaining unknown constant $\lambda_{3}$ is considered to be one more viscosity term or secondary viscosity term $\mu^{\prime}$ (Lamb 1945) in the case of solids. Thus the resistance due to strain rate is,

$$
\boldsymbol{P}_{\boldsymbol{d}}^{\prime}=\mu^{\prime} \frac{\mathrm{d}}{\mathrm{d} t}\left(\epsilon_{1}^{\prime}+\epsilon_{2}^{\prime}+\epsilon_{3}^{\prime}\right) \boldsymbol{I}+2 \mu \frac{\mathrm{d}}{\mathrm{d} t}\left(\left[\begin{array}{ccc}
\epsilon_{1}^{\prime} & 0 & 0 \\
0 & \epsilon_{2}^{\prime} & 0 \\
0 & 0 & \epsilon_{3}^{\prime}
\end{array}\right]-\frac{\left(\epsilon_{1}^{\prime}+\epsilon_{2}^{\prime}+\epsilon_{3}^{\prime}\right)}{3} \boldsymbol{I}\right)
$$

To evaluate the constants $\lambda_{1}, \lambda_{2}, \lambda_{3}, \lambda_{4}$, we consider only small strain conditions. At small strains the values of elastic constant are not affected by the material nonlinearity and instability which occur at higher loads. The values of $K$ and $G$ can be obtained experimentally and are standard for a given material. Similarly the value of $\mu$ can be calculated from the torsional vibration of a tube. The value of $\mu^{\prime}$ can be calculated from the longitudinal vibration of a thin wire of the material.

Considering that deformation is continuous under the action of strain and strain rate, the total resistance for the deformation can be determined by adding the resistance from strain and strain rate terms. Thus adding (33) and (35) we get,

$$
\begin{aligned}
\boldsymbol{P}^{\prime}= & \boldsymbol{P}_{\boldsymbol{e}}^{\prime}+\boldsymbol{P}_{\boldsymbol{d}}^{\prime} \\
= & \left(K+\mu^{\prime} \frac{\mathrm{d}}{\mathrm{d} t}\right)\left(\epsilon_{1}^{\prime}+\epsilon_{2}^{\prime}+\epsilon_{3}^{\prime}\right) \boldsymbol{I} \\
& +2\left(G+\mu \frac{\mathrm{d}}{\mathrm{d} t}\right)\left(\left[\begin{array}{ccc}
\epsilon_{1}^{\prime} & 0 & 0 \\
0 & \epsilon_{2}^{\prime} & 0 \\
0 & 0 & \epsilon_{3}^{\prime}
\end{array}\right]-\frac{\left(\epsilon_{1}^{\prime}+\epsilon_{2}^{\prime}+\epsilon_{3}^{\prime}\right)}{3} \boldsymbol{I}\right) .
\end{aligned}
$$


This expression holds good for infinitesimal strain conditions where volume change is negligible. But during large deformation, the volume does not remain constant (Bell 1990). In such cases the change in volume should be taken into consideration. Therefore the hydrostatic pressure should be expressed in terms of volumetric strain or, in other words, in terms of strain invariants. Thus for large deformation, the above equation is modified as,

$$
\begin{aligned}
\boldsymbol{P}^{\prime}= & \left(K+\mu^{\prime} \frac{\mathrm{d}}{\mathrm{d} t}\right)\left(S_{1}^{\prime}+S_{2}^{\prime}+S_{3}^{\prime}\right) \boldsymbol{I} \\
& +2\left(G+\mu \frac{\mathrm{d}}{\mathrm{d} t}\right)\left(\left[\begin{array}{ccc}
e_{1} & 0 & 0 \\
0 & e_{2} & 0 \\
0 & 0 & e_{3}
\end{array}\right]-\frac{S_{1}^{\prime}+S_{2}^{\prime}+S_{3}^{\prime}}{3} \boldsymbol{I}\right),
\end{aligned}
$$

where $e_{1}, e_{2}, e_{3}$ are the principal stretches as defined by Fung (1965) and $S_{1}^{\prime}, S_{2}^{\prime}$ and $S_{3}^{\prime}$ are the three invariants of the principal stretches,

$$
\begin{aligned}
& S_{1}^{\prime}=e_{1}+e_{2}+e_{3}, \\
& S_{2}^{\prime}=e_{1} e_{2}+e_{2} e_{3}+e_{3} e_{1}, \\
& S_{3}^{\prime}=e_{1} e_{2} e_{3} .
\end{aligned}
$$

To observe the material behaviour under external force, consider the elastic resistance part. The above equation reduces to,

$$
\boldsymbol{P}^{\prime}=(3 K-2 G)\left(\frac{S_{1}^{\prime}+S_{2}^{\prime}+S_{3}^{\prime}}{3}\right) \boldsymbol{I}+2 G\left(\begin{array}{ccc}
e_{1} & 0 & 0 \\
0 & e_{2} & 0 \\
0 & 0 & e_{3}
\end{array}\right)
$$

From the uniaxial tension test we get,

$$
\left[\begin{array}{ccc}
\sigma & 0 & 0 \\
0 & 0 & 0 \\
0 & 0 & 0
\end{array}\right]=(3 K-2 G)\left(\frac{S_{1}^{\prime}+S_{2}^{\prime}+S_{3}^{\prime}}{3}\right) \boldsymbol{I}+2 G\left[\begin{array}{ccc}
e_{1} & 0 & 0 \\
0 & e_{2} & 0 \\
0 & 0 & e_{3}
\end{array}\right],
$$

where $\sigma$ is the principal stress. In the case of a bar which is symmetric along the $z$-axis, $e_{2}=e_{3}$. Thus the change in volume is given by,

$$
S_{1}^{\prime}+S_{2}^{\prime}+S_{3}^{\prime}=e_{1}+2 e_{2}+e_{2}\left(2 e_{1}+e_{2}\right)+e_{1} e_{2}^{2} .
$$

By solving (39) we get,

$$
\sigma=2 G\left(e_{1}-e_{2}\right) .
$$

In the case of material instability as explained by Panovko \& Gubanova (1965), we get the relationship between the instability function $\left(1+e_{2}\right)^{2}$ and $\sigma / G$ as,

$$
\begin{aligned}
p & =A\left(1+e_{2}\right)^{2} \sigma \\
& =2 A\left(1+e_{2}\right)^{2} G\left(e_{1}-e_{2}\right), \\
\Rightarrow \quad p / 2 A G & =\left(1+e_{2}\right)^{2}\left(e_{1}-e_{2}\right) .
\end{aligned}
$$

Now, we can plot $e_{1}$ and $e_{2}$ as a function of $\sigma / G$. The value of $e_{1}$ can be calculated by assuming some negative values for $e_{2}$ and solving (41) using (40) for $\sigma / G$. These plots are as shown 
in figures 25 to 28 . In figure 25 a we can see that $e_{2}$ increases linearly with $\sigma / G$ at very low load as it is only a function of $S_{1}^{\prime}$. With increase in applied force, the curve shows nonlinear behaviour as the remaining two invariants of strain i.e. $S_{2}^{\prime}$ and $S_{3}^{\prime}$ increase their contributions. At very high applied force, it becomes flatten (figure 26a). That is, on the further increase in load, the increase in strain in lateral direction is very small. Thus we see reduction in cross sectional area but this does not go to zero as compared to our earlier observations. At the same time we observe that $e_{1}$ increases linearly with applied load (figure 25b) and remains linear at very high load also (figure 26b). From figure $25 \mathrm{c}$ and $26 \mathrm{c}$ we see that the strain ratio $e_{1} / e_{2}$ varies linearly with increase in load.

At the same time, the change in volume proportional to load confined to earlier deformation (figure 25d), later on further increase of load, the change in volume keeps gotting smaller (figure 26d) but does not go to zero as in earlier observations.

The instability function, behaves nonlinearly with axial strain (figure 27) but the amount of nonlinearity is small compared to earlier observations (figure 28).

Similar behaviour can be observed in the case of compressive loading. Figures 29 to 32 shows the plots for cases of compressive loading.

It is important to note that in both the tensile and compressive cases the graphs are not exact mirror images of each other at the $135^{\circ}$ axis line. The numerical values for each case are different for the given range of loading.

These graphs shows the continuous relation between stress and stretch and do not shows any sign of breaking. We also observe that the change in volume remains almost constant during large deformation.

We hypothesise that the breaking of the material therefore occurs at some critical load when a surface crack appears. The theoretical value for this breaking critical load for a material is approximately given by $E / \pi$ (Anderson 1995) based on surface energy consideration. But in practice this value is far lowe ouring to the presence of inhomogeneities.
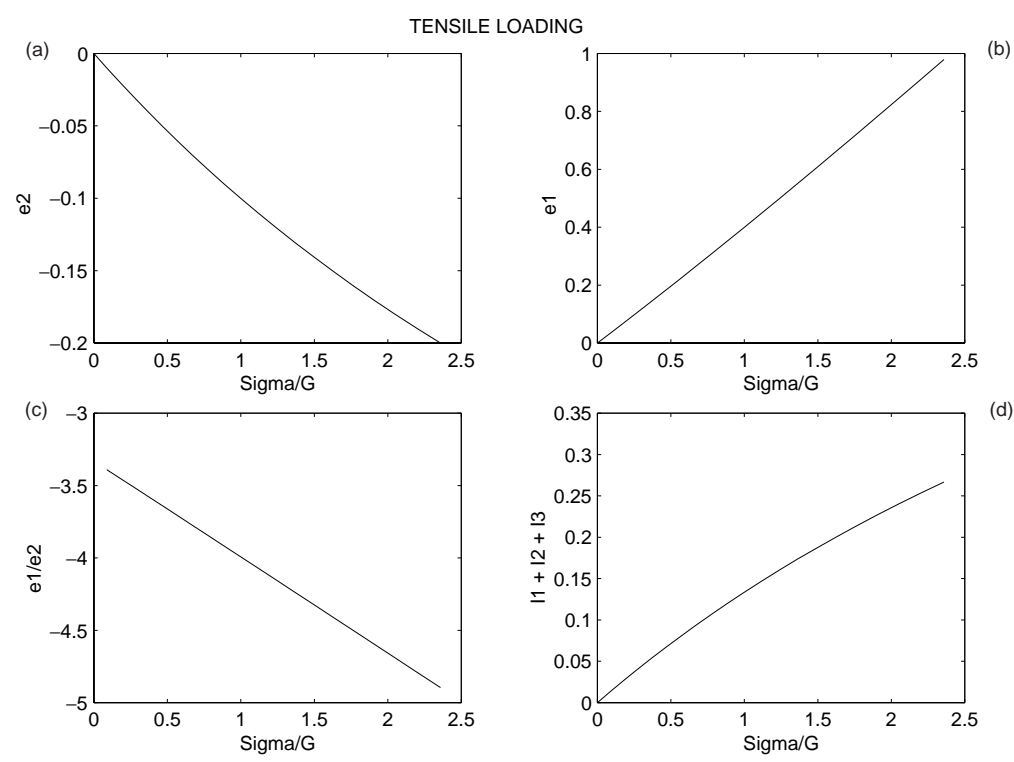

Figure 25. Behaviour of strain at small tensile load. (a) Second, and (b) first principal stretch; (c) Ratio of first and second principal stretch; (d) volumetric strain. 

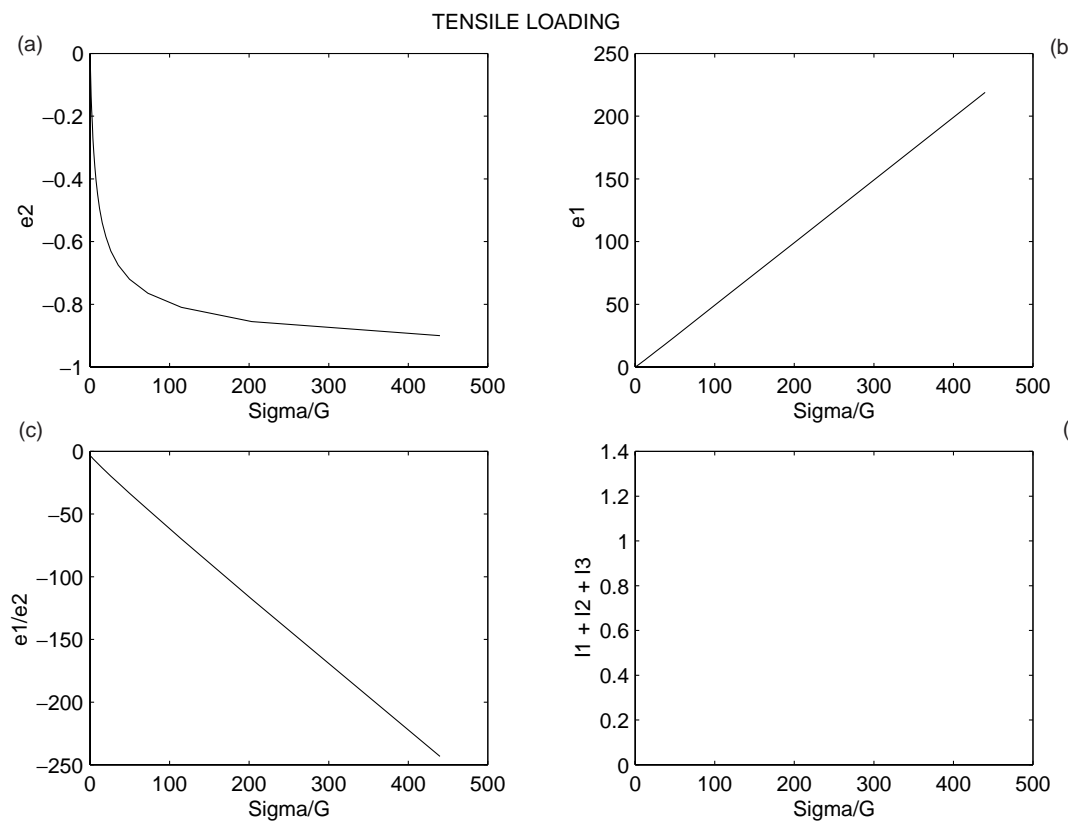

Figure 26. Behaviour of strain at higher tensile load. (a) Second, and (b) first principal stretch; (c) Ratio of first and second principal stretch; (d) volumetric strain.
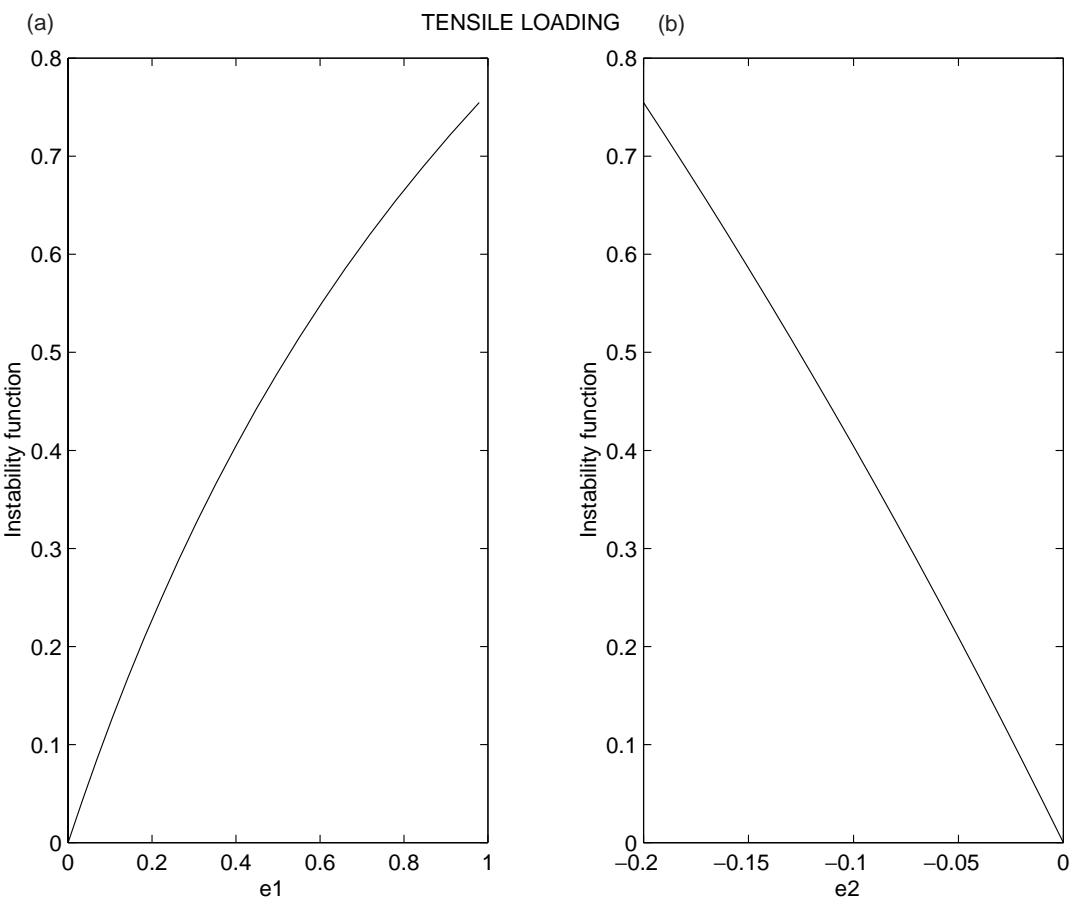

Figure 27. Behaviour of instability at small tensile load. (a) First and (b) second principal stretch vs instability function. 

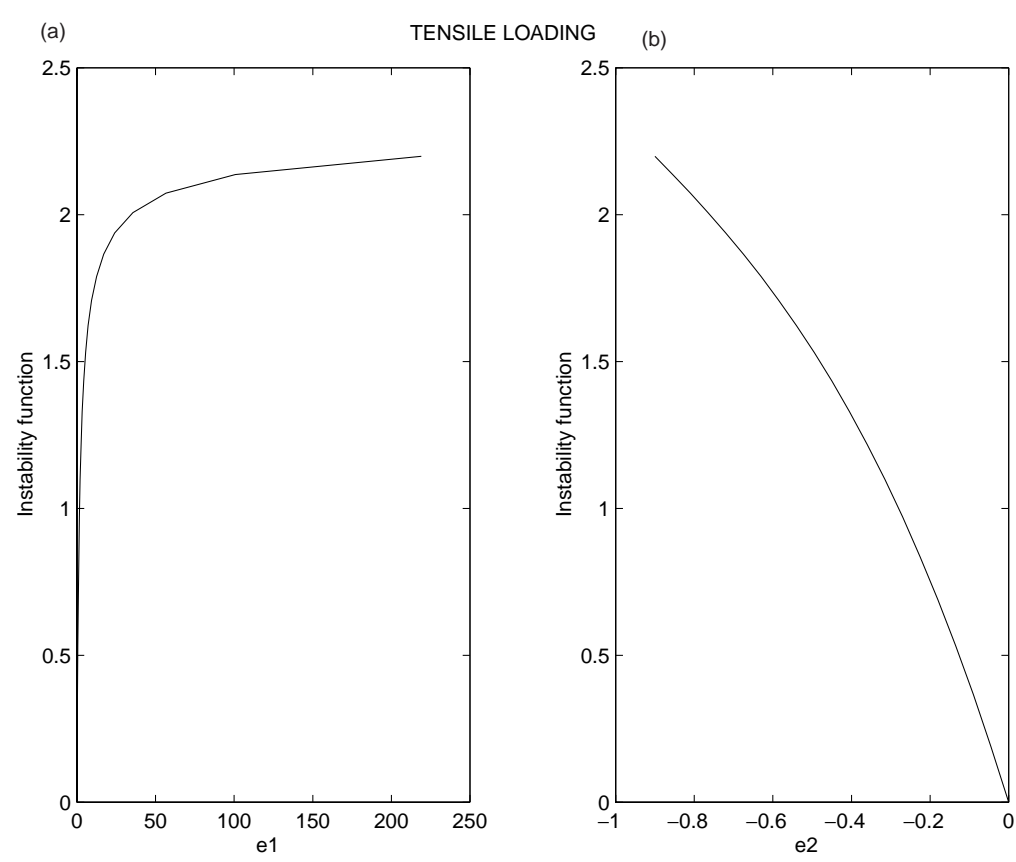

Figure 28. Behaviour of instability at higher tensile load. (a) First and (b) second principal stretch vs instability function.
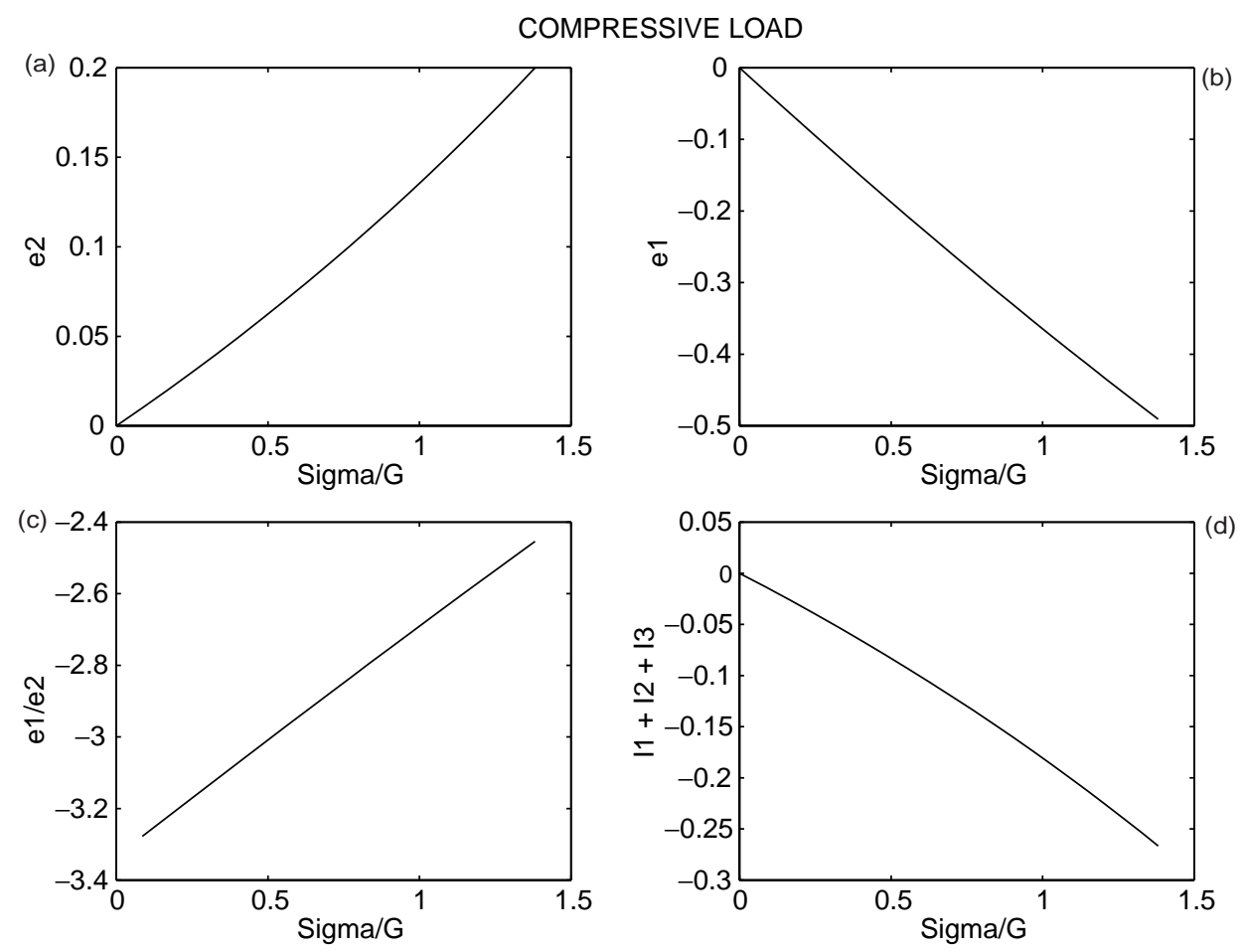

Figure 29. Behaviour of strain at small compressive load. (a) Second, and (b) first principal stretch; (c) Ratio of first and second principal stretch; (d) volumetric strain. 

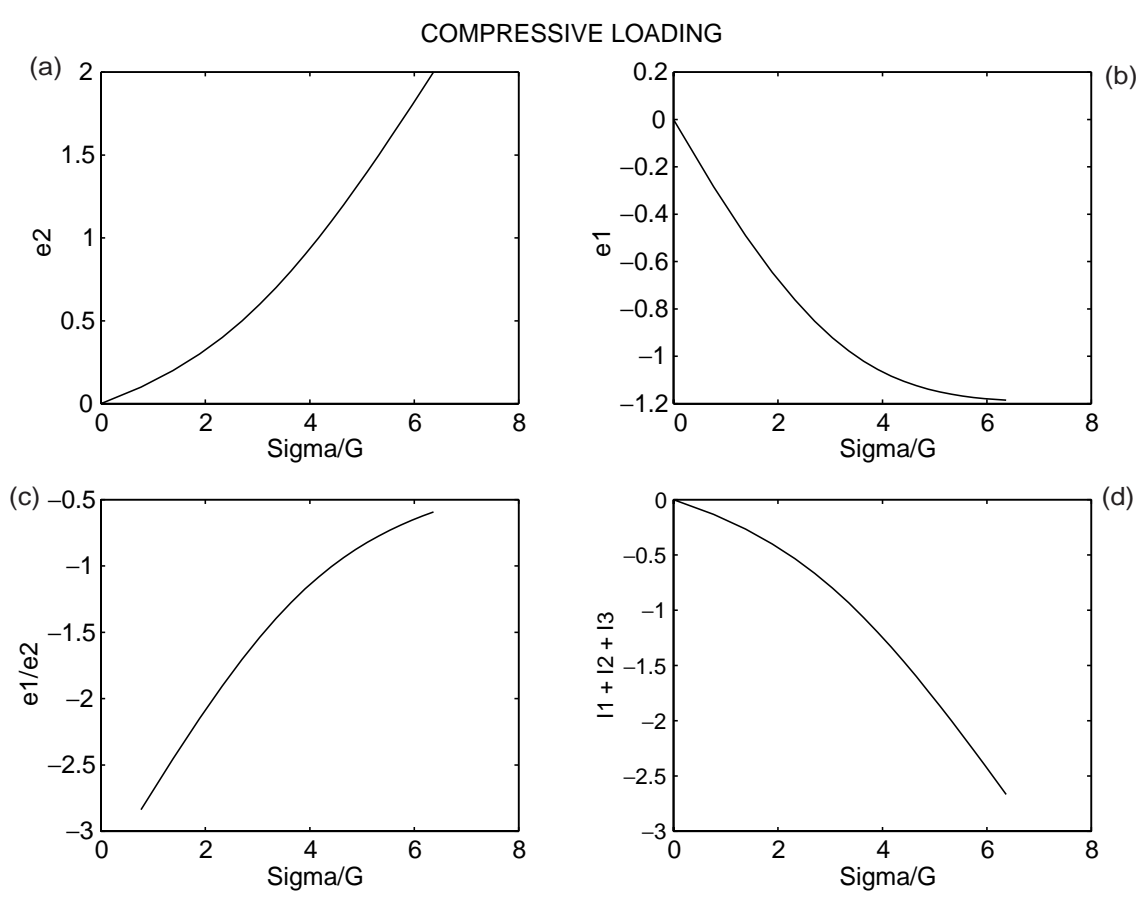

Figure 30. Behaviour of strain at higher compressive load. (a) Second and (b) first principal stretch; (c) Ratio of first and second principal stretch; (d) volumetric strain.
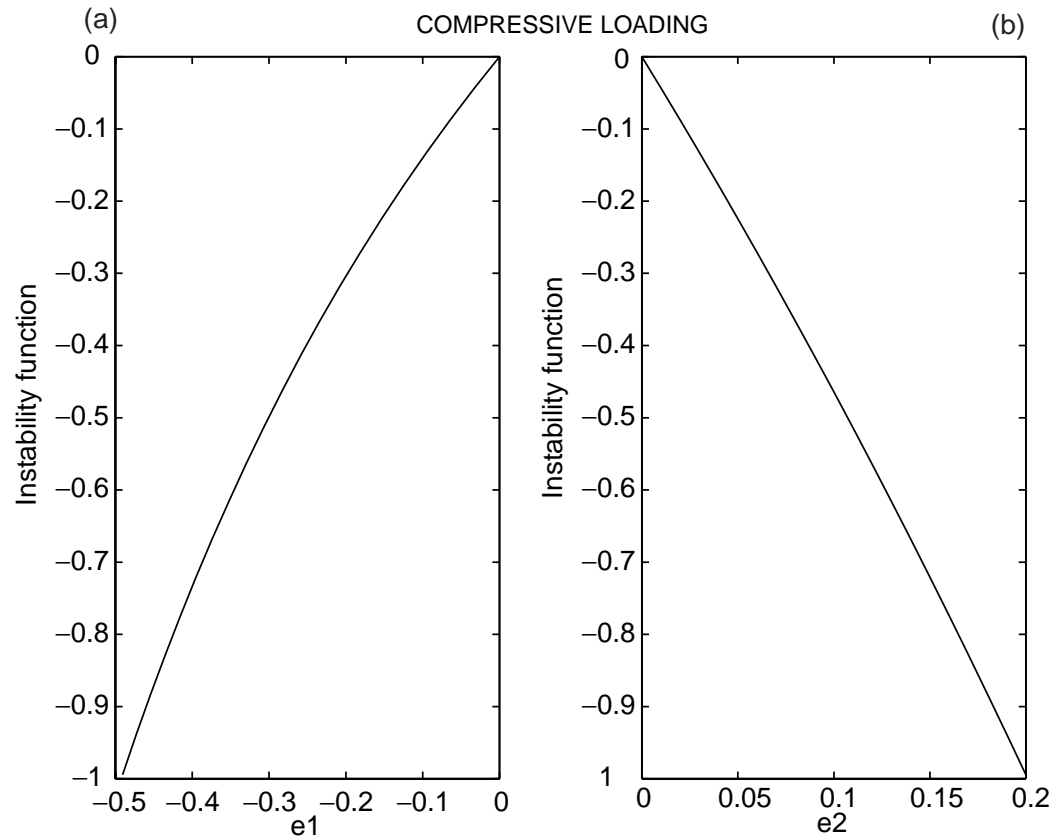

Figure 31. Behaviour of instability at small compressive load. (a) First and (b) second principal stretch vs instability function. 
(a)

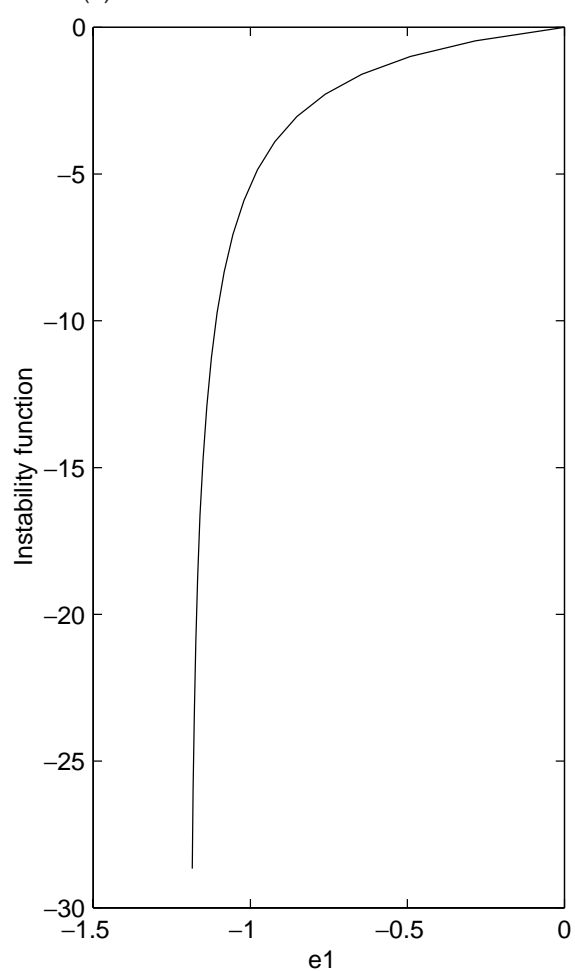

COMPRESSIVE LOADING

(b)

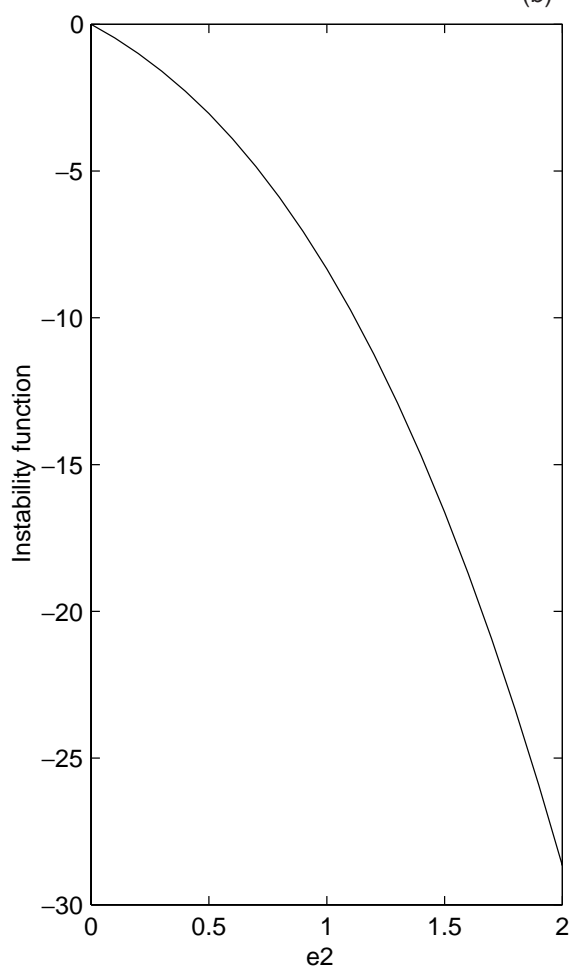

Figure 32. Behaviour of instability at higher compressive load. (a) First and (b) Second principal stretch vs instability function.

In the triaxial state of stress case, the stress acting on surface is given by total force applied on a cross-sectional area. Therefore the stresses acting on each surface $\boldsymbol{P}_{\boldsymbol{e}}^{\prime}$ of a infinitesimal tetrahedron is given by,

$$
\begin{aligned}
\boldsymbol{P}_{e}^{\prime} & =\left[\begin{array}{ccc}
\sigma_{1} /\left(1+e_{2}\right)\left(1+e_{3}\right) & 0 & 0 \\
0 & \sigma_{2} /\left(1+e_{3}\right)\left(1+e_{1}\right) & 0 \\
0 & 0 & \sigma_{3} /\left(1+e_{1}\right)\left(1+e_{2}\right)
\end{array}\right] \\
& =\frac{1}{\left(1+e_{1}\right)\left(1+e_{2}\right)\left(1+e_{3}\right)}\left[\begin{array}{ccc}
\sigma_{1}\left(1+e_{1}\right) & 0 & 0 \\
0 & \sigma_{2}\left(1+e_{2}\right) & 0 \\
0 & 0 & \sigma_{3}\left(1+e_{3}\right)
\end{array}\right] .
\end{aligned}
$$

Let $\mathcal{I}=S_{1}^{\prime}+S_{2}^{\prime}+S_{3}^{\prime}$ be the change in volume. Therefore the above equation can be written as,

$$
\boldsymbol{P}_{\boldsymbol{e}}^{\prime}=\frac{1}{1+\mathcal{I}}\left[\begin{array}{ccc}
\sigma_{1}\left(1+e_{1}\right) & 0 & 0 \\
0 & \sigma_{2}\left(1+e_{2}\right) & 0 \\
0 & 0 & \sigma_{3}\left(1+e_{3}\right)
\end{array}\right]
$$


Substituting (44) in (38) and writing each terms separately we get,

$$
\begin{aligned}
& \sigma_{1}=\left(1+e_{2}\right)\left(1+e_{3}\right)\left[(3 K-2 G) \frac{\mathcal{I}}{3}+2 G e_{1}\right], \\
& \sigma_{2}=\left(1+e_{3}\right)\left(1+e_{1}\right)\left[(3 K-2 G) \frac{\mathcal{I}}{3}+2 G e_{2}\right], \\
& \sigma_{3}=\left(1+e_{1}\right)\left(1+e_{2}\right)\left[(3 K-2 G) \frac{\mathcal{I}}{3}+2 G e_{3}\right] .
\end{aligned}
$$

From the above equations we can write the first, second and third invariants of the stress tensor in terms of the strain tensor and the volumetric strain as,

$$
\begin{aligned}
\Theta_{1}= & (3 K-2 G) \mathcal{I}+(2(3 K-2 G) \mathcal{I} / 3+2 G) S_{1}^{\prime} \\
& +((3 K-2 G) \mathcal{I} / 3+2 G) S_{2}^{\prime}+6 G S_{3}^{\prime}, \\
\Theta_{2}= & (1+\mathcal{I})\left[3(2 G-3 K)^{2}(\mathcal{I} / 3)^{2}\right. \\
& +(((2 G-3 K) \mathcal{I} / 3-4 G)(2 G-3 K) \mathcal{I} / 3) S_{1}^{\prime} \\
& \left.+4 G(G-(2 G-3 K) \mathcal{I} / 3) S_{2}^{\prime}+12 G^{2} S_{3}^{\prime}\right] \\
\Theta_{3}= & (1+\mathcal{I})^{2}\left[(3 K-2 G)^{3}(\mathcal{I} / 3)^{3}+2 G(3 K-2 G)^{2}(\mathcal{I} / 3)^{2} S_{1}^{\prime}\right. \\
& \left.+4 G^{2}(3 K-2 G)(\mathcal{I} / 3) S_{2}^{\prime}+8 G^{3} S_{3}^{\prime}\right]
\end{aligned}
$$

Thus by knowing strain and volumetric strain we can calculate the stress and stress invariants. Similarly we calculate strain and strain invariants by knowing stress and volumetric strain from (44) as,

$$
\begin{aligned}
& a^{\prime}=\frac{(1+\mathcal{I})(3 K-2 G) \mathcal{I} / 3-\sigma_{1}}{\sigma_{1}-2 G(1+\mathcal{I})}, \\
& b^{\prime}=\frac{(1+\mathcal{I})(3 K-2 G) \mathcal{I} / 3-\sigma_{2}}{\sigma_{2}-2 G(1+\mathcal{I})}, \\
& c^{\prime}=\frac{(1+\mathcal{I})(3 K-2 G) \mathcal{I} / 3-\sigma_{3}}{\sigma_{3}-2 G(1+\mathcal{I})}
\end{aligned}
$$

Now, we can write strain invariants in terms stress and volumetric strain as,

$$
\begin{aligned}
I_{1}^{\prime}= & \frac{(1+\mathcal{I})(3 K-2 G) \mathcal{I} / 3-\sigma_{1}}{\sigma_{1}-2 G(1+\mathcal{I})}+\frac{(1+\mathcal{I})(3 K-2 G) \mathcal{I} / 3-\sigma_{2}}{\sigma_{2}-2 G(1+\mathcal{I})} \\
& +\frac{(1+\mathcal{I})(3 K-2 G) \mathcal{I} / 3-\sigma_{3}}{\sigma_{3}-2 G(1+\mathcal{I})}
\end{aligned}
$$




$$
\begin{aligned}
I_{2}^{\prime}= & \frac{(1+\mathcal{I})(3 K-2 G) \mathcal{I} / 3-\sigma_{1}}{\sigma_{1}-2 G(1+\mathcal{I})} \cdot \frac{(1+\mathcal{I})(3 K-2 G) \mathcal{I} / 3-\sigma_{2}}{\sigma_{2}-2 G(1+\mathcal{I})} \\
& +\frac{(1+\mathcal{I})(3 K-2 G) \mathcal{I} / 3-\sigma_{2}}{\sigma_{2}-2 G(1+\mathcal{I})} \cdot \frac{(1+\mathcal{I})(3 K-2 G) \mathcal{I} / 3-\sigma_{3}}{\sigma_{3}-2 G(1+\mathcal{I})} \\
& +\frac{(1+\mathcal{I})(3 K-2 G) \mathcal{I} / 3-\sigma_{3}}{\sigma_{3}-2 G(1+\mathcal{I})} \cdot \frac{(1+\mathcal{I})(3 K-2 G) \mathcal{I} / 3-\sigma_{1}}{\sigma_{1}-2 G(1+\mathcal{I})} \\
I_{3}^{\prime}= & \frac{(1+\mathcal{I})(3 K-2 G) \mathcal{I} / 3-\sigma_{1}}{\sigma_{1}-2 G(1+\mathcal{I})} \cdot \frac{(1+\mathcal{I})(3 K-2 G) \mathcal{I} / 3-\sigma_{2}}{\sigma_{2}-2 G(1+\mathcal{I})} \\
& \frac{(1+\mathcal{I})(3 K-2 G) \mathcal{I} / 3-\sigma_{3}}{\sigma_{3}-2 G(1+\mathcal{I})} .
\end{aligned}
$$

\subsection{Final form of the equation}

During large deformation, the small strain tensors are not able to provide the complete geometrical interpretation. Fung (1965) used the concept of stretch to interpret large deformation as,

$$
e_{1}=1-\left(1-2 \epsilon_{1}\right)^{1 / 2},
$$

where he considered $\epsilon_{1}$ as a small strain component. However, here we treat it as a component of the Almansi strain tensor. After some simplification we see that both stretch and strain are of the same order.

Equation (37) gives the final form of the resistance to deformation in the principal coordinate system. Using the transformation matrix, and going back to the spatial coordinate system,

$$
\begin{aligned}
\boldsymbol{P} & =\boldsymbol{L}^{T} \boldsymbol{P}^{\prime} \boldsymbol{L} \\
& =\left(K+\mu^{\prime} \frac{\mathrm{d}}{\mathrm{d} t}\right)\left(S_{1}+S_{2}+S_{3}\right) \boldsymbol{I}+2\left(G+\mu \frac{\mathrm{d}}{\mathrm{d} t}\right)\left(\mathcal{E}-\frac{S_{1}+S_{2}+S_{3}}{3} \boldsymbol{I}\right),
\end{aligned}
$$

where $\mathcal{E}$ is the stretch tensor in the spatial coordinate system and $S_{1}, S_{2}, S_{3}$ are the corresponding invariants.

$$
\begin{aligned}
\mathcal{E} & =\boldsymbol{I}-\left(\boldsymbol{I}-2 \boldsymbol{E}^{*}\right)^{1 / 2}, \\
S_{1} & =\mathcal{E}_{i i}, \\
S_{2} & =-\frac{1}{2}\left(\mathcal{E}_{i j} \mathcal{E}_{i j}-\mathcal{E}_{i i} \mathcal{E}_{j j}\right), \\
S_{3} & =\operatorname{det}(\mathcal{E}) .
\end{aligned}
$$

Now substituting $\boldsymbol{P}$ in (10), the law of balance of linear momentum in Eulerian coordinate and simplifying, we get the final form of the equation of motion as,

$$
\begin{aligned}
\rho \frac{D \dot{\mathbf{U}}}{D t}= & \rho \mathbf{B}+\nabla^{T}\left[\left(3 K-2 G+\left(3 \mu^{\prime}-2 \mu\right) \frac{\mathrm{d}}{\mathrm{d} t}\right)\left(\frac{S_{1}+S_{2}+S_{3}}{3}\right)\right] \\
& +\left[\nabla \cdot\left(2\left(G+\mu \frac{\mathrm{d}}{\mathrm{d} t}\right) \mathcal{E}\right)\right]^{T} .
\end{aligned}
$$


We observe that the above equation has only three variables, i.e. three displacements along three directions. The density term can be expressed in the form of volumetric strain as we have seen during the earlier formulation. The pressure term $p$ does not appear in this formulation. Thus there is no one more equation, the continuity equation, as we did earlier. The change in material density during the deformation automatically takes care of the mass conservation contrary to that assumed in the case of fluid. Fluid is expected to deform large by under shear load and therefore the effect of volume change is separately related to hydrostatic pressure during deformation. Thus an additional equation is required to take into account the conservation of mass as a supplementary to the Navier-Stokes equation for fluids. This however is avoided in our final formulation above. As discussed earlier, the four unknown material constants $K, G, \mu^{\prime}$ and $\mu$ can be obtained from simple measurements. The equations are applicable from solids to fluids and therefore could form the basis for discussing plasticity of solids by including their microscopic inhomogeneities as well as for discussing turbulence in fluids by including their microscopic structures as found in flowing fluids.

\section{Conclusions}

The finalised field equation is obtained in the Eulerian coordinate system using Almansi strain and Cauchy stress tensors without any major assumptions. The developed relation shows that deformation occurs due to continuous contribution from strain and strain rate terms. Under the action of load, material behaves like a compressible fluid and change in volume occurs due to changes in strain invariants. The addition of the elastic effect in the formulation considers residual stresses and spring-back actions in the case of elasto-plastic deformation. Elastic unloading naturally occurs in our formulation. Resistance to non-elastic deformation comes from gradients of rate of deformation. The approach strengthens the theory put forth by microscopic modelling of plastic deformation that local gradients caused by defects and microscopic inhomogeneities determine the plastic behaviour of materials. Instabilities during tension tests are brought out by the model. The role of inclusions and microscopic inhomogeneities in plastic flow can also be observed. The model explains the classical understanding of the high strength of materials drawn into thin wires. Bauschinger effect, material anisotropy, yield point phenomenon, effect of stress inhomogeneities, and fracture are easily explained by the model. It also helps to understand material strengthening, ductility and failure phenomena.

\section{Appendix A}

Consider an equilibrium system with the forces $\sigma_{x}, \sigma_{y}$ and $\sigma_{z}$ acting along the $x, y$ and $z$ direction respectively in the principal coordinate system. Let $\epsilon_{x}, \epsilon_{y}$ and $\epsilon_{z}$ be the corresponding strains as shown in figure A1. Now consider the situation where a body undergoes deformation in all three directions. We can write stresses acting at a point in equilibrium as,

$$
\begin{aligned}
& \sigma_{x}=E \epsilon_{x}\left(1+\epsilon_{y}-v\left(\epsilon_{x}+\epsilon_{z}\right)\right)\left(1+\epsilon_{z}-v\left(\epsilon_{x}+\epsilon_{y}\right)\right), \\
& \sigma_{y}=E \epsilon_{y}\left(1+\epsilon_{z}-v\left(\epsilon_{y}+\epsilon_{x}\right)\right)\left(1+\epsilon_{x}-v\left(\epsilon_{y}+\epsilon_{z}\right)\right), \\
& \sigma_{z}=E \epsilon_{z}\left(1+\epsilon_{x}-v\left(\epsilon_{z}+\epsilon_{y}\right)\right)\left(1+\epsilon_{y}-v\left(\epsilon_{z}+\epsilon_{x}\right)\right) .
\end{aligned}
$$




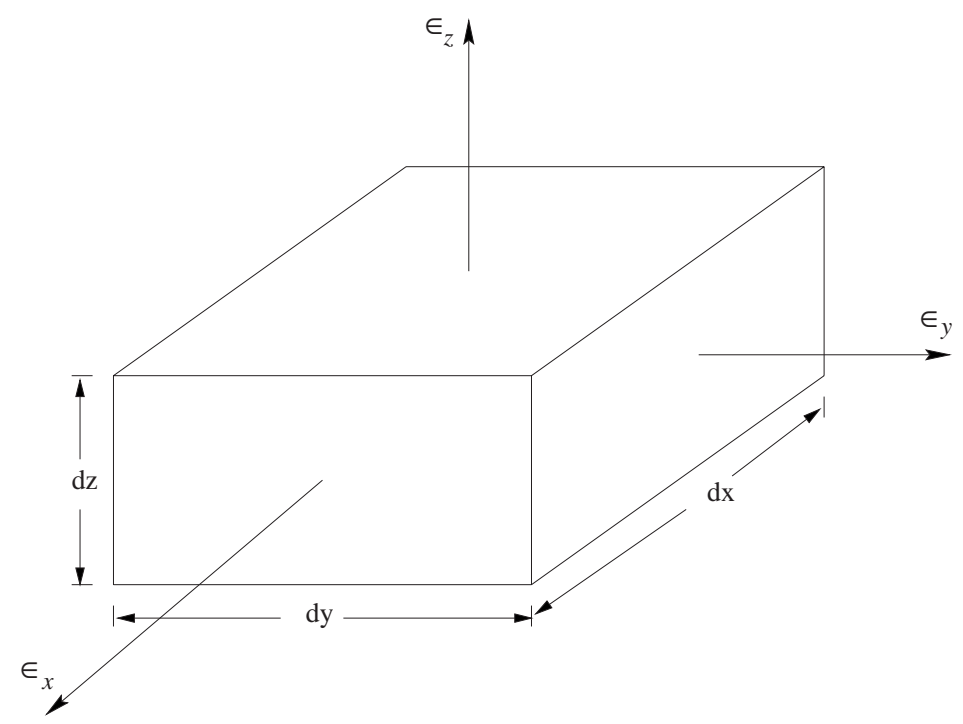

Figure A1. Strains in a body under equilibrium.

Let $\epsilon_{1}, \epsilon_{2}$ and $\epsilon_{3}$ be the first, second and third invariants of strain. i.e.,

$$
\begin{aligned}
& \epsilon_{1}=\epsilon_{x}+\epsilon_{y}+\epsilon_{z}, \\
& \epsilon_{2}=\epsilon_{x} \epsilon_{y}+\epsilon_{y} \epsilon_{z}+\epsilon_{z} \epsilon_{x}, \\
& \epsilon_{3}=\epsilon_{x} \epsilon_{y} \epsilon_{z} .
\end{aligned}
$$

Adding equations for $\sigma_{x}, \sigma_{y}$ and $\sigma_{z}$ and rearranging the terms, we get the first invariant of stress $\Theta_{1}^{\prime}$ as,

$$
\Theta_{1}^{\prime}=E\left[\epsilon_{1}-2 v \epsilon_{1}^{2}+v^{2} \epsilon_{1}^{3}-2(1+v) \epsilon_{2}+2 v(1-v) \epsilon_{1} \epsilon_{2}+3(1+v)^{2} \epsilon_{3}\right],
$$

where

$$
\Theta_{1}^{\prime}=\sigma_{x}+\sigma_{y}+\sigma_{z}
$$

The second invariant of stress $\Theta_{2}$ can be obtained by,

$$
\Theta_{2}^{\prime}=E^{2}\left(\sigma_{x} \sigma_{y}+\sigma_{y} \sigma_{z}+\sigma_{z} \sigma_{x}\right),
$$

substituting from (A1) in the above equation, expanding and rearranging the terms, we get,

$$
\begin{aligned}
\Theta_{2}^{\prime}= & E^{2}\left[\epsilon_{2}-(1+v) \epsilon_{1} \epsilon_{2}-3 v(1-v) \epsilon_{1}^{2} \epsilon_{2}+v^{2}(1-v) \epsilon_{1}^{3} \epsilon_{2}-v^{3} \epsilon_{1}^{4} \epsilon_{2}\right. \\
& +3(1+v)^{4} \epsilon_{3}^{2}+(1+v)^{2} \epsilon_{2}^{2}+3(1+v)(1-2 v) \epsilon_{1} \epsilon_{3}-2 v(1+v)^{2} \epsilon_{1} \epsilon_{2}^{2} \\
& -3 v(2-v)(1+v) \epsilon_{1}^{3} \epsilon_{3}+v^{2}(1+v)^{2} \epsilon_{1}^{2} \epsilon_{2}^{2}+3 v^{2}(1+v) \epsilon_{1}^{3} \epsilon_{3} \\
& \left.+2(1+v)^{3} \epsilon_{2} \epsilon_{3}-4 v(1+v)^{3} \epsilon_{1} \epsilon_{2} \epsilon_{3}+3(1+v) \epsilon_{3}\right] .
\end{aligned}
$$


Similarly, the third invariant of stress $\Theta_{3}^{\prime}$ can be determined,

$$
\begin{aligned}
\Theta_{3}^{\prime}= & \sigma_{x} \sigma_{y} \sigma_{z} \\
= & E^{3} \epsilon_{3}\left[1+(1-2 v) \epsilon_{1}+v(v-2)\left(\epsilon_{1}^{2}-2 \epsilon_{2}\right)-v\left(1-v+v^{2}\right)\left(\epsilon_{1} \epsilon_{2}-3 \epsilon_{3}\right)\right. \\
& \left.+\left(1-2 v+3 v^{2}\right) \epsilon_{3}+\left(1+3 v^{2}-2 v^{3}\right) \epsilon_{2}+v^{2}\left(\epsilon_{1}^{3}-3 \epsilon_{1} \epsilon_{2}+3 \epsilon_{3}\right)\right]^{2}
\end{aligned}
$$

The elements of the matrix $C$ which appear in (23) of $\S 3$ while developing the instability criteria are given by,

$$
\begin{gathered}
\sigma_{x} / \epsilon_{x}=-v \epsilon_{x}\left(1+\epsilon_{z}-v\left(\epsilon_{x}+\epsilon_{y}\right)\right)-v \epsilon_{x}\left(1+\epsilon_{y}-v\left(\epsilon_{x}+\epsilon_{z}\right)\right) \\
+\left(1+\epsilon_{z}-v\left(\epsilon_{x}+\epsilon_{y}\right)\right)\left(1+\epsilon_{y}-v\left(\epsilon_{x}+\epsilon_{z}\right)\right), \\
\sigma_{x} / \epsilon_{y}=\epsilon_{x}\left(1+\epsilon_{z}-v\left(\epsilon_{x}+\epsilon_{y}\right)\right)-v \epsilon_{x}\left(1+\epsilon_{y}-v\left(\epsilon_{x}+\epsilon_{z}\right)\right), \\
\sigma_{x} / \epsilon_{z}=-v \epsilon_{x}\left(1+\epsilon_{z}-v\left(\epsilon_{x}+\epsilon_{y}\right)\right)+\epsilon_{x}\left(1+\epsilon_{y}-v\left(\epsilon_{x}+\epsilon_{z}\right)\right), \\
\sigma_{y} / \epsilon_{x}=\epsilon_{y}\left(1+\epsilon_{z}-v\left(\epsilon_{x}+\epsilon_{y}\right)\right)-v \epsilon_{y}\left(1+\epsilon_{x}-v\left(\epsilon_{y}+\epsilon_{y}\right)\right), \\
\sigma_{y} / \epsilon_{y}=-v \epsilon_{y}\left(1+\epsilon_{z}-v\left(\epsilon_{x}+\epsilon_{y}\right)\right)-v \epsilon_{y}\left(1+\epsilon_{x}-v\left(\epsilon_{y}+\epsilon_{z}\right)\right), \\
\quad+\left(1+\epsilon_{z}-v\left(\epsilon_{x}+\epsilon_{y}\right)\right)\left(1+\epsilon_{y}-v\left(\epsilon_{x}+\epsilon_{z}\right)\right), \\
\sigma_{y} / \epsilon_{z}=-v \epsilon_{y}\left(1+\epsilon_{z}-v\left(\epsilon_{x}+\epsilon_{y}\right)\right)+\epsilon_{y}\left(1+\epsilon_{x}-v\left(\epsilon_{y}+\epsilon_{z}\right)\right), \\
\sigma_{z} / \epsilon_{x}=\epsilon_{z}\left(1+\epsilon_{y}-v\left(\epsilon_{x}+\epsilon_{z}\right)\right)-v \epsilon_{z}\left(1+\epsilon_{x}-v\left(\epsilon_{y}+\epsilon_{z}\right)\right), \\
\sigma_{z} / \epsilon_{y}=-v \epsilon_{z}\left(1+\epsilon_{y}-v\left(\epsilon_{x}+\epsilon_{z}\right)\right)+\epsilon_{z}\left(1+\epsilon_{x}-v\left(\epsilon_{y}+\epsilon_{z}\right)\right), \\
\sigma_{z} / \epsilon_{z}=-v \epsilon_{z}\left(1+\epsilon_{y}-v\left(\epsilon_{x}+\epsilon_{z}\right)\right)-v \epsilon_{z}\left(1+\epsilon_{x}-v\left(\epsilon_{y}+\epsilon_{z}\right)\right) \\
\quad+\left(1+\epsilon_{y}-v\left(\epsilon_{x}+\epsilon_{z}\right)\right)\left(1+\epsilon_{x}-v\left(\epsilon_{y}+\epsilon_{z}\right)\right) .
\end{gathered}
$$

\section{References}

Acharya A 2001 A model of crystal plasticity based on the theory of continuously distributed dislocations. J. Mech. Phys. Solids 49: 761-784

Acharya A, Beaudoin A J 2000 Grain-size effect in viscoplastic polycrystals at moderate strains. $J$. Mech. Phys. Solids 48: 2213-2230

Agah-Tehrani A, Lee E H, Mallet R L, Onat E T 1987 The theory of elasto-plastic deformation at finite strain with induced anisotropy modeled as combined isotropic-kinematic hardening. J. Mech. Phys. Solids 35: 519-539

Anderson T L 1995 Fracture mechanics: Fundamental and applications (Boca Raton, FL: CRC Press)

Anjaneyulu P 1999 Towards elasto-plastic modelling, using modified Navier-Stokes equation. M E thesis, Dept of Mechanical Engineering, Indian Institute of Science, Bangalore

ASTM 1985 Metals - Mechanical testing; elevated and low temperature tests, metallography. Annual book of ASTM standards, (Philadelphia, PA:) Am. Soc. Testing Mater. 03.01, E-8

Atluri S N 1984 On constitutive relations at finite strain; Hypo-elasticity and elasto-plasticity with isotropic or kinematic hardening. Comput. Meth. Appl. Mech. Engng 43: 137-171

Backman M E 1964 From the relation between stress and finite elastic and plastic strains under impulsive loading. J. Appl. Phys. 35: 2524-2533 
Barbe F, Decker L, Jeulin D, Cailletaud G 2001 Intergranular and intragranular behaviour of polycrystalline aggregates. Part 1: F.E. model. Int. J. Plasticity 17: 513-536

Batdorf S B, Budiansky B 1949 A mathematical theory of slip based on the concept of slip. NACA TN 1871, April

Bazant Z P 1984 Microplane model for strain controlled inelastic behavior. In Mechanics of engineering materials (eds) C S Desai, R H Gallagher (London: John Wiley) pp 15-59

Bell J F 1990 Material objectivity in an experimentally based incremental theory of large finite plastic strains. Int. J. Plasticity 6: 293

Bertram A 1998 An alternative approach to finite plasticity based on material isomorphisms. Int. J. Plasticity 52: 353-374

Bigoni D, Loret B, Radi E 2000 Localization of deformation in plane elastioplastic solids with anisotropic elasticity. J. Mech. Phys. Solids 48: 1441-1466

Bodner S R, Parton Y 1975 Constitutive equations for elasto-viscoplastic strain hardening materials. J. Appl. Mech. 42: 385-389

Braess H, Wriggers P 2000 Arbitrary Lagrangian Eulerian finite element analysis of free surface flow. Comput. Meth. Appl. Mech. Engng 190: 95-109

Budiansky B, Wu T T 1962 Theoretical prediction of plastic strains in polycrystals. Proc. 4th US Natl. Congr. Appl. Mech. (New York: Am. Soc. Mech. Eng.) pp 1175-1185

Casey J, Naghdi P M 1981 On the characterization of strain-hardening in plasticity. J. Appl. Mech. 48: 285-296

Casey J, Naghdi P M 1983 A remark on the defination of hardening, softening and perfectly plastic behavior. Acta Mech. 48: 91-94

Chakrabarty J 1998 Theory of plasticity (Singapore:McGraw-Hill)

Chen C, Lu T J 2000 A phenomenological framework of constitutive modelling for incompressible and compressible elasto-plastic solids. Int. J. Solids Struct. 37: 7769-7786

Dafalias Y F 1983 Corotational rates for kinematic hardening at large plastic deformation. J. Appl. Mech. 50: 561-565

Dafalias Y F 1984 The plastic spin concept and a simple illustration of its role in plastic transformations. Mech. Mater. 3: 223-233

Dafalias Y F 1985 The plastic spin. J. Appl. Mech. 52: 865-871

Dienes J K 1979 On the analysis of rotation and stress rate in deformation bodies. Acta Mech. 32: 217-232

Dienes J K 1986 A discussion of material rotation and stress rate. Acta Mech. 65: 1-11

Drucker D C 1960 Plasticity. Proc. 1st Symp. Naval Structural Mechanics (Oxford: Pergamon) pp 407-455

Drucker D C 1988 Conventional and unconventional plastic response and representation. Appl. Mech. Rev. 41: 151-168

Fung Y C 1965 Foundation of solid mechanics (Englewood Cliffs, NJ: Prentice Hall)

Ghosh S, Suresh R 1996 R-s adapted arbitrary Lagrangian-Eulerian finite element method for metal forming problems with strain localization. Int. J. Numer. Meth. Engng. 86: 3247-3272

Green A E, Naghdi P M 1965 A general theory of an elastic-plastic continuum. Arch. Rational Mech. Anal. 18: 251-281

Green A E, Naghdi P M 1966 A thermodynamic development of elasto-plastic continuum. In Proc. IUTAM Symp. on Irreversible Aspects of Continuum Mechanics and Transfer of Physical Characteristics in Moving Fluids (eds) H Parker, L I Sedov (New York: Springer-Verlag) pp 117-131

Griffith A A 1921 The phenomena of rapture of flow in solids. Philos. Trans. R. Soc. London A221: $163-198$

Haber R B 1984 A mixed Eulerian-Lagrangian displacement model for large deformation analysis in solid mechanics. Comput. Meth. Appl. Mech. Engng. 43: 277-292

Hill R 1950 The mathematical theory of plasticity (London: Oxford University Press)

Hill R 1965 Continuum micromechanics of elastoplastic polycrystals. J. Mech. Phys. Solids 13: 89-102 
Hill R 1966 Generalized constitutive relations for incremental deformation of metal crystals by multislip. J. Mech. Phys. Solids 14: 95-102

Hill R 1994 Classical plasticity: a retrospective view and a new propasal. J. Mech. Phys. Solids 42: $1803-1826$

Huetink J, Vreede P T, Van Der Lugt J 1990 Progress in mixed Eulerian-Lagrangian finite element simulation of forming processes. Int. J. Numer. Meth. Engng. 30: 1441-1457

Hutchinson J W 1970 Elastic-plastic behaviour of polycrystalline metals and composites. Proc. $R$. Soc. London A319: 247-272

Koiter W T 1953 Stress-strain relations, uniqueness and variational theorms for elastic-plastic materials with a singular yield surface. Q. Appl. Math. 11: 350-354

Kröner E 1960 Allgemeine Kontinuumstheorie der Versetzungen und Eigenspannungen. Arch. Rational Mech. Anal. 4: 273-334

Lamb R H 1945 Hydrodynamics (New York: Dover)

Lazan B J 1968 Damping of materials and members in strutural mechanics (Oxford: Pergamon)

Lee D, Zaverl F Jr 1978 A generalized strain rate dependent constitutive equation for anisotropic materials. Acta Metall. 26: 1771-1780

Lee D, Zaverl F Jr 1979 A description of history dependent plastic flow behavior of anisotropic metals. J. Engng. Mater. Technol. Trans. ASME 101: 59-67

Lee E H 1969 Elastic-plastic deformation at finite strains. J. Appl. Mech. 36: 1-6

Lee E H, Liu D T 1967 Finite strain elastic-plastic theory with application to plane-wave analysis. $J$. Appl. Phys. 38: 19-27

Lee E H, Mallett R L, Wertheimer T B 1983 Stress analysis for anisotropic hardening in finitedeformation plasticity. J. Appl. Mech. 50: 554-560

Loret B 1983 On the effects of plastic rotation in the finite deformation of anisotropic elastoplastic materials. Mech. Mater. 2: 287-304

Malvern L E 1969 Introduction to the mechanics of continuous medium (Englewood Cliffs, NJ: Prentice Hall)

Naghdi P M 1960 Stress-strain relations in plasticity and thermoplasticity. Proc. 2nd Symp. Naval Structural Mechanics (Oxford: Pergamon) pp 121-167

Naghdi P M, Trapp J A 1975a The significance of formulating plasticity theory with reference to loading surfaces in strain space. Int. J. Engng. Sci. 13: 785-797

Naghdi P M, Trapp J A 1975b Restriction on constitutave equations of finitely deformed elastic-plastic materials. Q. J. Mech. Appl. Math. 28: 25-46

Nemat-Nasser S 1983 On plastic flow of crystalline solids and geomaterials. J. Appl. Mech. 50: 11141126

Panovko Y G, Gubanova I I 1965 Stability and oscillations of elastic systems-paradoxes, fallacies, and new concepts (New York: Consultants Bureau)

Prager W 1955 The theory of plasticity: A survey of recent achivements (John Clyton Lecture). Proc. Inst. Mech. Eng. 169: 41-57

Prager W 1961 Introduction to mechanics of continua (Boston, MA: Ginn)

Prager W, Hodge P G Jr 1951 Theory of perfectly plastic solids (New York: John Wiley)

Qiu Y P, Weng G J 1992 A theory of plasticity for porous materials and particle-reinforced composites. J. Appl. Mech. ASME 59: 261-268

Qiu Y P, Weng G J 1995 An energy approach to the plasticity of a two-phase composite containing aligned inclusion. J. Appl. Mech. ASME 62: 1039-1046

Rice J R 1976 The localization of plastic deformation. In Theoretical and applied mechanics (ed.) W T Koiter (Amsterdam: North Holland)

Rubin M B 1996 On the treatment of elastic deformation in finite elastic-viscoplastic theory. Int. J. Plasticity 12: 951-965

Seth B R 1963 Elastic-plastic transition in shells and tubes under pressure. Z. Angew. Math. Mech. 43: 345-351

Seth B R 1964 Elastic-plastic transition in torsion. Z. Angew. Math. Mech. 44: 229-233 
Seth B R 1970 Transition conditions: The yield condition. Int. J. Non-Linear Mech. 5: 279-285

Stouffer D C, Bodner S R 1979. A constitutive model for the deformation induced anisotropic plastic flow of metals. Int. J. Engng. Sci. 17: 757-764

Taylor G I, Elam C F 1923 The distortion of an aluminium crystal during tensile test. Proc. R. Soc. London A102: 643-667

Taylor G I, Elam C F 1925 The plastic extension and fracture of aluminium crystals. Proc. R. Soc. London A108: 28-51

Taylor G I, Elam C F 1926 The distortion of iron crystals. Proc. R. Soc. London A112: 337-361.

Timoshenko S P 1986 Strength of materials, Part-2: Advanced theory and problems (Delhi: CBS)

Timoshenko S P, Goodier J N 1970 Theory of elasticity Int. edn (Singapore: McGraw-Hill)

Valanis K C, 1971 A theory of viscoplasticity without a yield surface. Part I General theory. Arch. Mech. 23: 517-533

Valanis K C 1975 On the foundation of the endochronic theory of viscoplasticity. Arch. Mech. 27: $857-868$

White F M 1994 Fluid mechanics (Singapore: McGraw Hill)

Zienkiewicz O C, Godbole P N, 1974 Flow of plastic and visco-plastic solids with special referance to extrusion and forming processes. Int. J. Numer. Meth. Engng. 8: 3-16.

Zienkiewicz O C, Godbole P N 1975 Viscous incompressible flow with special reference to nonnewtonian (plastic) fluids. In Finite elements in fluids (eds) Gallagher et al (Chichester: Wiley Interscience) vol. 1, pp 25-55 